\title{
ROLE OF THE GALANIN N-TERMINAL FRAGMENT (1- 15) IN ANHEDONIA: INVOLVEMENT OF THE DOPAMINERGIC MESOLIMBIC SYSTEM
}

Carmelo Millón ${ }^{1,2^{\star}}$, Antonio Flores-Burgess ${ }^{1}$, Belén Gago ${ }^{1}$, Francisco Alén², Laura Orio², Laura García-Durán¹, José Angel Narváez¹, Kjell Fuxe $^{3}$, Luis Santín ${ }^{4} \&$ Zaida Díaz-Cabiale ${ }^{1}$.

1.Universidad de Málaga, Instituto de Investigación Biomédica de Málaga, Facultad de Medicina, Campus de Teatinos s/n, 29071 Málaga, Spain.

2. Departamento de Psicobiología y Metodología en Ciencias del Comportamiento, Facultad de Psicología, Universidad Complutense de Madrid, Spain.

3. Department of Neuroscience, Karolinska Institute, Stockholm, Sweden.

4.Universidad de Málaga, Instituto de Investigación Biomédica de Málaga, Facultad de Psicología, Campus de Teatinos s/n, 29071 Málaga, Spain.

*Corresponding author:

Carmelo Millón Peñuela, PhD

Departamento de Fisiología

Facultad de Medicina

Universidad de Málaga

Campus de Teatinos s/n. 29080 Málaga, Spain

E-mail: carmelomp@uma.es

Short Title: Galanin (1-15) and anhedonia

Keywords: Galanin, Galanin (1-15), Depression, Anhedonia, Reward circuit, Dopamine.

Number of words in the abstract: 251

Number of words in the main text: 4424

Number of figures: 7

Number of tables: 2

Number of supplemental information: 1 


\section{ABSTRACT}

BACKGROUND: Anhedonia is a core feature of depressive disorders. The Galanin N-terminal fragment (1-15) [GAL(1-15)] plays a role in mood regulation since it induces depression and anxiogenic-like effects in rats. In this study, we analysed GAL(1-15) actions in anhedonic-like behaviours in rats using operant and non-operant tests and the areas involved with these effects.

METHODS: GAL(1-15) effects were analysed in saccharin self-administration, sucrose preference, novelty-suppressed feeding and female urine sniffing tests. The areas involved in GAL(1-15)-mediated effects were studied with positron emission tomography (PET) for in vivo imaging, and we analysed the ventral tegmental area (VTA) and nucleus accumbens (NAc). GAL(1-15) had effects on the mRNA expression of the dopamine transporters Dat and Vmat2; the C-Fos gene; the dopamine receptors D1, D2, D3, D5; and the GALRs (GALR1 and GALR2).

RESULTS: $\mathrm{GAL}(1-15)$ at a concentration of $3 \mathrm{nmol}$ induced a strong anhedonia-like phenotype in all tests. The involvement of GALR2 was demonstrated with the GALR2 antagonist M871 (3 nmol). The 18F-FDG PET images indicated the action of $\mathrm{GAL}(1-15)$ over several nuclei of the limbic system. GAL(1-15)-mediated effects also involved changes in the expression of Dat, Vmat2, D3 receptor and GALR in VTA as well as the expression of C-Fos, D1, D2 and D3 and TH immunoreactivity in the NAc.

CONCLUSIONS: Our results indicated that GAL(1-15) exerts strong anhedoniclike effects and that this effect was accompanied by changes in the dopaminergic mesolimbic system. These results may provide a basis for the development of novel therapeutic strategies using $\mathrm{GAL}(1-15)$ analogues for the treatment of depression and reward-related diseases. 
Millón et al., 2018

Journal of Psychopharmacology

\section{INTRODUCTION}

In 2016, the World Bank Group and the World Health Organization reported that mental disorders currently account for $30 \%$ of the nonfatal disease burden worldwide and that global cost is expected to reach $\$ 6$ trillion by 2030 (Marquez and Saxena, 2016). Major depression is the most frequent mood disorder, with a lifetime prevalence that has been reported to range from $7 \%$ to $21 \%$ (Albert et al., 2011). It is associated with substantial functional impairment, diminished quality of life, increased burden both for patients and caregivers, and a higher risk of mortality.

Although the underlying mechanisms have not yet been clearly defined in the last decade, the importance of the roles of neuropeptides, including Galanin (GAL), and/or their receptors in the treatment of stress-related mood disorders is becoming increasingly apparent (Kormos and Gaszner, 2013; Genders et al., 2018).

GAL is a neuropeptide (Tatemoto et al., 1983) that is widely distributed in neurons within the central nervous system (CNS) (Jacobowitz et al., 2004). Three GAL receptor (GALR1-3) subtypes with high affinities for GAL have been cloned (Branchek et al., 2000; Mitsukawa et al., 2008). GALR1 and GALR3 mainly activate the inhibitory $\mathrm{G}$ proteins $\mathrm{Gi} / \mathrm{Go}$ while GALR2 mainly couples to Gq/G11 to mediate excitatory signalling (Branchek et al., 2000). GAL and its receptors evoke depression-related and anxiety-like behaviours (Juhasz et al., 2014). GAL-overexpressing mice and rodents in which GAL was infused either intraventricularly (i.c.v) or into the ventral tegmental area showed an increase in immobility during a forced swimming test (FST) that was indicative of 
depression-like behaviour (Kuteeva et al., 2005; Kuteeva et al., 2007; Weiss et al., 1998). Additionally, in a genetic rat model of depression, the Flinders Sensitive Line, upregulation of the GAL receptor binding sites was found in the dorsal raphe nucleus, which has been linked to high immobility in the FST (Bellido et al., 2002). However, intraperitoneal (i.p.) injections of GALR2 agonists exhibited antidepressant-like effects and two non-selective GALR agonists, galnon and galmic, decreased immobility times in the FST (Bartfai et al., 2004; Lu et al., 2005). These discrepancies are attributed to the different physiological roles of the GAL receptor subtypes (Swanson et al., 2005; Kuteeva et al., 2008b; Barr et al., 2006). Thus, the activation of GALR1 and GALR3 results in a depression-like behaviour while stimulation of GALR2 leads to antidepressant-like effects (Bartfai et al., 2004; Lu et al., 2005; Kuteeva et al., 2008a). Recently, it has also been described that, in humans, the GAL gene and its receptor GALR3 are differentially methylated and expressed in brains of major depression subjects in a region- and sex-specific manner (Barde et al., 2016).

Not only GAL but also N-terminal fragments similar to $\mathrm{GAL}(1-15)$ are active in the CNS (Hedlund and Fuxe, 1996; Diaz-Cabiale et al., 2005; Diaz-Cabiale et al., 2007; Diaz-Cabiale et al., 2010). Recently, we demonstrated that GAL(1-15) induces strong depression-related behaviour in rats and these effects were significantly stronger than the ones induced by GAL (Millon et al., 2015). We analysed GAL(1-15)-mediated effects in two tests developed to screen monoamine-based antidepressant drugs: the FST and the tail suspension test (TST). Both assess the response to an inescapable stressor, provoking despairbased behaviour/immobility and, in these tests, GAL(1-15) induced depression- 
like behaviour since significant increases in immobility were observed (Millon et al., 2015). We demonstrated that GALR1-GALR2 heteroreceptor complexes in the dorsal hippocampus and DR, areas rich in $\mathrm{GAL}(1-15)$ binding sites (Hedlund et al., 1992) were involved in these effects (Millon et al., 2015) (Borroto-Escuela et al., 2014).

Moreover, GAL(1-15) also induced an anxiogenic-like effect in the open field and the light/dark test (Millon et al., 2015). Since, in animal models, anxiety can by considered an element of the depression, the fact that $\operatorname{GAL}(1-15)$ induced a strong depression and anxiogenic-like effects in all the tests indicated a potential role for GAL(1-15) in mood disorders, especially in depression.

However, the role of $\mathrm{GAL}(1-15)$ in an endophenotype that represents a key symptom of depression, such as reward-related and anhedonic behaviour, is still unknown. Anhedonia is considered to be a core feature of major depressive disorders as, for example, the Diagnostic and Statistical Manual of Mental Disorders, fifth edition (DSM-V) requires that either a depressed mood or anhedonia be present to propose this diagnosis. Furthermore, the lack of reactivity and anhedonia are key diagnostic criteria for the $D S M-V$ melancholic subtype of major depression, and the presence of anhedonia has been shown to be predictive of an antidepressant response (Gorwood, 2008).

The purpose of the present study was to assess the role of intracerebroventricular (i.c.v.) $\mathrm{GAL}(1-15)$ in anhedonic behaviour using a widely accepted approach to assess reward seeking behaviour in operant and nonoperant tests: saccharin self-administration, sucrose preference, noveltysuppressed feeding and female urine sniffing tests. Moreover, the involvement 
of GALR2 in the effects induced by GAL(1-15) were analysed with the selective GALR2 antagonist M871. To investigate the areas involved in GAL(1-15)mediated effects, positron emission tomography (PET) for in vivo imaging of metabolic processes was performed. We also analysed transcriptional changes in the ventral tegmental area (VTA) and nucleus accumbens (NAc), which are essential nuclei in reward processes, and the effect of GAL(1-15) on the mRNA expression of dopamine transporters Dat and Vmat2; the C-Fos gene; the dopamine receptors $D 1, D 2, D 3, D 5$; and the GALRs (GALR1 and GALR2).

\section{MATERIALS AND METHODS}

\section{Animals}

Male Sprague Dawley rats $(225-250 \mathrm{~g})$ were obtained from Criffa and maintained in a humidity- and temperature-controlled room. All animal experimentation was conducted in accordance with the University of Málaga Guidelines for the Care and Use of Laboratory Animals.

Detailed descriptions are available in the supplemental information on the animal controlled-conditions, surgical preparation and i.c.v. injections.

\section{Saccharin Self-administration}

Training was achieved using a modification of the protocol reported by (Alen et al., 2009). Briefly, rats were placed on a water restriction schedule for 2-4 days to facilitate training of lever pressing. The rats were trained to self-administer saccharin $0.2 \%(\mathrm{w} / \mathrm{v})$ in 30 -min daily sessions for two weeks on a fixed ratio 1 schedule of reinforcement in which each response resulted in the delivery of 0.1 $\mathrm{ml}$ of fluid. At this point, saccharin self-administration training continued until the 
animals reached stable baseline responding. During the test sessions, the responses on the active lever and number of saccharin reinforcements were recorded (see the supplemental information for details).

Three sets of experiments were conducted in the saccharin self-administration test. In the first set of experiments, a dose-response curve of $\mathrm{GAL}(1-15)$ was generated. For this, groups of rats received i.c.v. 1 nmol or 3 nmol GAL(1-15) or vehicle $15 \mathrm{~min}$ before the test. In the second set of experiments, the effects in saccharin self-administration of GAL and GAL(1-15) were compared. Groups of rats received i.c.v. $3 \mathrm{nmol}$ GAL, $3 \mathrm{nmol} \mathrm{GAL}(1-15)$ or vehicle $15 \mathrm{~min}$ before the test. In the last set of experiments, groups of rats received i.c.v. $3 \mathrm{nmol}$ GAL(115) was combined with GALR2 antagonist $3 \mathrm{nmol}$ M871 $15 \mathrm{~min}$ before the measures to study the role of GALR2. The doses employed and the injection protocol were based on those of previous studies (Millon et al., 2015).

The general schema of the experimental design is shown in Figure $1 \mathrm{~A}$.

\section{Sucrose Preference Test}

Anhedonia was assessed using the sucrose preference test (SPT), performed as described previously (Marco et al., 2017) with minor modifications (see the supplemental information for details). Briefly, on the testing day, rats were allowed free access to two-bottles: one containing $1 \%(w / v)$ sucrose solution and the other containing tap water. After 2, 8 and $24 \mathrm{~h}$, the bottles were weighed to calculate the sucrose intake $(\mathrm{g} / \mathrm{kg})$ and sucrose preference (sucrose preference=sucrose consumption /(water + sucrose consumption) $\times 100)$, which reflected the rats' anhedonia levels. 
Millón et al., 2018 Journal of Psychopharmacology

In the experiment, groups of rats received i.c.v. 3 nmol GAL(1-15) or vehicle, based on a previous study (Millón et al., 2015), and the sucrose intake and sucrose preference were measured at 2, 8 and $24 \mathrm{~h}$ after injection.

The general schema of the experimental design is shown in Figure 1B.

\section{Novelty Suppressed feeding}

The NSF test was carried out during the $10 \mathrm{~min}$ period as previously described (Pascual-Brazo et al., 2012) with some modifications (see the supplemental information for details). Briefly, all rats were deprived of food for $24 \mathrm{~h}$ before the test. The testing apparatus consisted of a plastic box $(60 \times 40 \times 20 \mathrm{~cm})$ with a single pellet of food in the centre of the box on white circular filter paper. In the experiment, groups of rats received i.c.v. $3 \mathrm{nmol}$ GAL(1-15) or vehicle $15 \mathrm{~min}$ before the test, based on a previous study (Millón et al., 2015), and the latency of the first feeding episode was recorded.

\section{Female Urine Sniffing Test}

The female urine sniffing test (FUST) was conducted as described previously (Malkesman et al., 2012) (see the supplemental information for details). Briefly, the test had three phases: a single exposure to the cotton tip dipped in sterile water; an interval during which no applicator was presented to the animal; and a single exposure to a cotton tip infused with fresh urine or water, during which the time spent sniffing was measured.

The animals receiving $3 \mathrm{nmol}$ GAL(1-15) or vehicle were injected 15 min before exposure to the urine or water. 
Millón et al., 2018

Journal of Psychopharmacology

The general schema of the experimental design of NSF and FUST is shown in Figure 1B.

\section{PET studies}

The ${ }^{18} \mathrm{~F}-\mathrm{FDG}$ PET imaging was performed at Unidad de Imagen Molecular, CIMES, Spain in a dedicated small animal Philips MOSAIC tomograph (Cleveland, OH, USA) system as previously described (Prieto et al., 2011). Animals were administered icv with CSFa and, after 3-4 days, with Gal(1-15) (3 $\mathrm{nmol}$ ). The doses employed and the injection protocol were based on previous works (Millon et al., 2015). Immediately after the treatment, the ${ }^{18}$ F-FDG (43.6 \pm $5.5 \mathrm{MBq}$ in $0.3 \mathrm{ml}$ of $0.9 \% \mathrm{NaCl}$ ) was injected through the tail vein. After an awake uptake period of 25 minutes to ensure the incorporation of ${ }^{18} \mathrm{~F}-\mathrm{FDG}$ during drug-induced behavioural effects (Prieto et al., 2011), the animals were anaesthetized and placed prone on the PET scanner bed to perform static acquisition for $30 \mathrm{~min}$ at three different timepoints $(30,60$ and $90 \mathrm{~min})$ after tracer administration (see the supplemental information for details).

\section{Statistical analysis of PET images}

Differences in ${ }^{18} \mathrm{~F}-\mathrm{FDG}$ uptake between aCSF- and GAL[1-15]-injected animals were assessed using software package SPM8 under the general lineal model framework and whole brain contrast analysis. A t-test was performed for each timepoint (30, 60 and $90 \mathrm{~min})$, and the differences between the groups were represented by a map of $\mathrm{t}$-statistics. The contrast images were yielded based on a voxel-level height threshold of $p<0.01$ (uncorrected) and a cluster-extend threshold of 100 voxels. Resultant clusters are shown on a SAMIT rat RM template in Paxinos and Watson space. 


\section{Genes expression in VTA and NAc}

Rats from NSF and FUST experiments (Figure 1B) were euthanized by decapitation $1 \mathrm{~h}$ after a single i.c.v. administration of $\mathrm{GAL}(1-15) 3 \mathrm{nmol}$ or vehicle and the brains were rapidly removed and frozen until use. The dose and time selected were based by previous works (Millón et al., 2015; García-Perez et al., 2016). The nuclei dissections were conducted as described (GarciaPerez et al., 2016) with modifications (see supplemental information for details).

RNA isolation and quantitative real-time polymerase chain reaction analysis

The procedure to perform RNA isolation and real-time polymerase chain reactions (RT-PCRs) was described previously (Millon et al., 2015; Millon et al., 2017a; Flores-Burgess et al., 2017) (see the supplemental information for details).

The primer sequences used to evaluate the mRNA expression levels of the genes Gapdh, C-Fos, Dat, Vmat2, D1, D2, D3, D5, GALR1 and GALR2 are shown in Table S1.

\section{Tyrosine Hydroxylase immunohistochemistry}

Rats from the NSF and FUST experiment (Figure 1B) were perfused transcardially 90 min after a single i.c.v. administration of $3 \mathrm{nmol} G A L(1-15)$ or vehicle. The $\mathrm{TH}$ immunohistochemistry was conducted in NAc and $\mathrm{CPu}$ with modifications as previously described (Cadoni et al., 2017) (see the supplemental information for details).

\section{Double immunofluorescence}


The procedures have been used previously (Millon et al., 2015). Primary antibodies directed to GALR2 (rabbit polyclonal, Alomone Lab, 1/250) and GALR1 (goat polyclonal, Santa Cruz Biotechnology INC, EEUU, 1:250) were used (see supplemental information for details). Double immunolabelling was performed in sections of the striatum and VTA of rats without treatment (Figure S1).

\section{Statistical Analysis}

Data are presented as the mean \pm standard error of the mean, and sample numbers $(n)$ are indicated in figure legends. All data were analysed using GraphPad PRISM 6.0 (GraphPad Software). For comparing two experimental conditions, Student's unpaired t-tests were performed. Otherwise, one-way or two-way analysis of variance (ANOVA) followed by Fisher's least significant difference (LSD) comparison post-test was performed. Differences were considered significant at $P<0.05\left({ }^{*} P<0.05,{ }^{* *} P<0.01,{ }^{* * *} P<0.001\right)$.

\section{RESULTS}

\section{Behavioural Studies}

\section{GAL(1-15) induced an anhedonic-like effect.}

Effects of $G A L(1-15)$ in the saccharin self-administration test

$\mathrm{GAL}(1-15)$ at a concentration of $3 \mathrm{nmol}$ significantly decreased the number of reinforcements of saccharin self-administration (one-way ANOVA, $F_{2,26}=5.743$, $p<0.01$, Fisher's LSD post hoc test: $p<0.01$ ) by $43 \%$ (Figure $2 A$ ) fifteen minutes after administration. GAL(1-15) at a concentration of 1 nmol lacked the effect on the number of saccharin reinforcements. 
In the comparison between $\mathrm{GAL}$ and $\mathrm{GAL}(1-15)$ in the saccharin selfadministration test, one-way ANOVA revealed a significant difference between the N-terminal fragment GAL(1-15) and GAL. GAL(1-15) significantly reduced the number of saccharin reinforcements compared with GAL (one-way ANOVA, $F_{2,28}=6.068, p<0.01$, Fisher's LSD post hoc test: $p<0.01$ ) (Figure $2 B$ ). GAL lacked an effect on the number of saccharin reinforcements compared with the control group (Figure 2B).

GALR2 antagonist M871 blocked GAL(1-15)-mediated effects in the saccharin self-administration test. The GALR2 antagonist M871 significantly blocked the decrease in the number of saccharin reinforcements (one-way ANOVA, $F_{3,28}=3.449, p<0.05$, Fisher's LSD post hoc test: $\left.p<0.05\right)$ induced by $\operatorname{GAL}(1-15)$ administration (Figure 2C). M871 alone at the dose of $3 \mathrm{nmol}$ lacked an effect on the number of saccharin reinforcements.

Effects of $G A L(1-15)$ in the sucrose preference test (SPT)

GAL(1-15) at a concentration of $3 \mathrm{nmol}$ decreased the sucrose intake (two-way ANOVA (group $x$ time), $F_{2,26}=4.8836, p<0.05$ ) at 8 hours (Fisher's LSD post hoc test $p<0.05$ ) and 24 hours (Fisher's LSD post hoc test $p<0.01$ ) after administration (Figure 3A). Moreover, i.c.v. injection of $3 \mathrm{nmol}$ GAL(1-15) induced a not significant decrease in sucrose preference (two-way ANOVA (Group), $\left.F_{1,13}=4.039, p=0.0656\right)$ at $2 \mathrm{~h}, 8 \mathrm{~h}$ and $24 \mathrm{~h}$ (Figure 3B).

Regarding water intake (Table S2), no differences (two-way ANOVA (Time x Group), $\left.F_{2,26}=0.2915, p=0.7494\right)$ were found at any timepoint after $G A L(1-15)$ i.c.v. administration.

Effects of GAL(1-15) in the novelty suppressed feeding (NSF) test 
In the NSF, GAL(1-15) at the dose of $3 \mathrm{nmol}$ significantly increase the latency to eat $\left(t_{15}=5.147, p<0.001 ;\right.$ Figure $\left.4 A\right)$ by $80 \%$.

Effects of GAL(1-15) in the female urine sniffing test (FUST)

In this test, the overall one-way ANOVA revealed a significant difference between the sniffing duration of water cotton and female urine cotton (one-way ANOVA, $F_{2,23}=14.39, p<0.001$, Fisher's LSD post hoc test: $p<0.001$ urine-CSFa; $\mathrm{p}<0.01$ urine-GAL(1-15); Figure 4B). Moreover, $3 \mathrm{nmol}$ GAL(1-15) induced a significant decrease in sniffing duration over urine cotton to compare with the urine-CSFa group (one-way ANOVA, $F_{2,23}=14.39$, $p<0.001$, Fisher's LSD post hoc test $\mathrm{p}<0.05$; Figure $4 \mathrm{~B})$.

No differences were found between the water-CSFa and water-GAL(1-15) groups in sniffing duration $\left(t_{10}=1.480, p=0.084\right.$; Figure $3 C$. $)$

\section{PET Studies}

The GAL(1-15) treatment induced a significant decrease in $\left[{ }^{18} \mathrm{~F}\right] \mathrm{FDG}$ uptake in the hippocampus (bilateral) and thalamus at $30 \mathrm{~min}$. This reduction was maintained in both nuclei at $60 \mathrm{~min}$ and $90 \mathrm{~min}$ (Figure 5). In addition, a reduced uptake was also observed in the striatum (caudate putamen and external globus pallidum) at $30 \mathrm{~min}$. Moreover, the administration of the GAL fragment induced an increase in $\left[{ }^{18} \mathrm{~F}\right] \mathrm{FDG}$ uptake after $30 \mathrm{~min}$ and $60 \mathrm{~min}$ in the prefrontal cortex and in the piriform cortex after 60 min (Figure 5).

\section{Neurochemical Studies}


GAL(1-15) affects the mRNA expression of the dopamine transporters Dat and Vmat2 and of C-Fos in the ventral tegmental area (VTA) and the accumbens nucleus (NAc).

As shown in Figure 6, GAL(1-15) at a dose of $3 \mathrm{nmol}$ produced a significant decrease in the mRNA levels of Dat $\left(t_{6}=1.988, p<0.05\right.$; Figure $\left.6 A\right)$ and Vmat2 $\left(t_{6}=2.480, p<0.05 ;\right.$ Figure 6B) in VTA 1 hour after its administration. However, GAL(1-15) did not affect C-Fos mRNA expression in the nucleus (Figure 6C). In the NAc, 3 nmol i.c.v. $\mathrm{GAL}(1-15)$ induced a significant decrease in the expression of C-Fos mRNA ( $t_{7}=2.329, p<0.05$; Figure 6D) 1 hour after administration.

Additionally, C-Fos mRNA expression was measured in other regions, such as the dentate gyrus in the hippocampus, where GAL(1-15) produced a significant increase compared with the control group (mRNA expression: Control: $\left.1.39 \pm 0.2 ; \mathrm{GAL}(1-15): 2.60 \pm 0.2 ; \mathrm{t}_{6}=3.352, \mathrm{p}<0.01\right)$.

GAL(1-15) affects the mRNA expression of dopamine receptors in the ventral tegmental area (VTA) and the accumbens nucleus (NAc)

In the VTA, the administration of GAL(1-15) induced a significant increase in the mRNA expression of $D 31$ hour after injection $\left(t_{7}=1.981, p<0.05\right.$; Figure $\left.7 C\right)$. No differences were found in $D 1, D 2$ and $D 5$ receptor expression after $G A L(1-15)$ administration in the VTA (Figure 7A,B, and D).

As shown in the second part of Figure $7, \operatorname{GAL}(1-15) 3$ nmol significantly increased the mRNA expression of $D 1 \quad\left(t_{7}=2.019, p<0.05 ;\right.$ Figure $\left.7 E\right), D 2$ $\left(t_{7}=2.008, p<0.05 ;\right.$ Figure $\left.7 F\right)$ and $D 3\left(t_{6}=3.045, p<0.05\right.$ Figure $\left.7 G\right)$ in the NAc, 
1 hour after its administration. GAL(1-15) lacked an effect on mRNA expression of $D 5$ compared with the control group in the NAc.

\section{GAL(1-15) effects in mRNA expression of Galanin receptors in the Ventral Tegmental Area (VTA) and Accumbens nucleus (NAc).}

Using double immunolabelling, we observed a colocation of GALR1 and GALR2 immunoreactivity in the nerve cells of the VTA (Figure S1A), in both the core (NAcC; Figure S1B) and shell (NAcSh; Figure S1C) of the NAc and in the dorsal striatum (caudate putamen; CPu) (Figure S1D).

The administration of GAL(1-15) modified GALR2 and GALR1 expression in the VTA, producing a significant increase in GALR2 mRNA levels $\left(t_{7}=1.970\right.$, $\mathrm{p}<0.05$; Table 1) and a slight increase in $G A L R 1$ expression $\left(\mathrm{t}_{7}=0.980, \mathrm{p}=0.17\right.$; Table 1), suggesting the involvement of GALR in GAL(1-15)-mediated effects.

\section{GAL(1-15) decreased TH immunoreactivity in the striatum.}

As shown in Table 2, i.c.v. administration of $\mathrm{GAL}(1-15)$ reduced $\mathrm{TH}$ immunoreactivity in the striatum. GAL(1-15) induced a significant decrease in TH immunoreactivity in the NAc core $\left(t_{4}=2.694, p<0.05\right.$; Table 2) 90 min after its administration. Moreover, the injection of $\mathrm{GAL}(1-15)$ induced a slight decrease in $\mathrm{TH}$ immunoreactivity in the NAc Shell. In the dorsal striatum (CPu); the same response pattern was observed in that $\mathrm{GAL}(1-15)$ produced a slight reduction in TH immunoreactivity 90 min after its injection (Table 2).

\section{DISCUSSION}


In the present study, we demonstrated for the first time that GAL(1-15) induced a strong anhedonia-like phenotype in non-operant and operant models: the sucrose preference test (SPT), the novelty suppressed feeding (NSF) test, the female urine sniffing test (FUST) and the operant saccharin self-administration test. GALR2 was involved in this effect since the specific GALR2 antagonist M871 blocked GAL(1-15) mediated action in saccharin self-administration.

${ }^{18}$ F-FDG PET images demonstrated that GAL(1-15) induced modifications in FDG uptake in vivo in limbic nuclei, such as the hippocampus, the thalamus, striatum, the prefrontal cortex and the piriform cortex. Importantly, the mechanism of the GAL(1-15) behaviour-mediated effect was accompanied by changes in the VTA and NAc, which are the key nuclei of the dopaminergic mesolimbic circuit and are critical for the neurobiological bases of anhedonia (Der-Avakian and Markou, 2012). Changes in the expression of the dopamine transporters DAT and Vmat2 and in the D3 and Galanin receptors in the VTA were observed. Changes in the immediate-early gene C-Fos and in the D1, D2 and D3 receptors in NAc were also observed. The relevance of the mesolimbic circuit as a target for GAL(1-15) was also supported by the effect of GAL(1-15) on the $\mathrm{TH}$ immunoreactivity in the striatum.

In the operant self-administration models used as a tool for the study of rewardseeking motivated behaviour (Sanchis-Segura and Spanagel, 2006; Ettenberg, 2009), GAL(1-15) induced a strong reduction in the number of reinforcements in saccharin self-administration, suggesting that GAL(1-15) induced a loss of motivational behaviour induced by an artificial reinforcer. 
In this work, we also studied the differential role of $G A L(1-15)$ and $G A L$ in reward-seeking motivated behaviour using the saccharin self-administration paradigm. GAL(1-15), but not GAL, induced a strong reduction in the number of reinforcements, suggesting that only $\mathrm{GAL}(1-15)$ reduces the motivation to consume the reward. We have previously described a different action of GAL and GAL(1-15) in behaviour functions (Millon et al., 2015; Millon et al., 2016; Millon et al., 2017b; Millon et al., 2017a). GAL(1-15) induces depression-related and anxiogenic-like effects in rats, and these effects were significantly stronger than the one induced by GAL (Millon et al., 2015). Furthermore, GAL(1-15) produced a strong reduction in the alcohol intake and preference in a model of voluntary alcohol intake, showing a different role than that of the complete GAL molecule (Millon et al., 2017a). The differential action between GAL and GAL(1-15) was observed not only in behavioural functions but also in central cardiovascular regulation (Diaz-Cabiale et al., 2005; Diaz-Cabiale et al., 2010).

Therefore, the fact that the effects of $\operatorname{GAL}(1-15)$ in the saccharin selfadministration were different than the effects induced by GAL validated and extended the view of a specific role of $\mathrm{GAL}(1-15)$ in reward-related and anhedonic behaviour.

We demonstrated that GALR2 is involved in the anhedonia-like effects induced by $\mathrm{GAL}(1-15)$ since the administration of the specific GALR2 antagonist M871 blocked the effects of $\operatorname{GAL}(1-15)$. The formation of heterodimers between GALR1/GALR2 has been proposed to be the main mechanism of action of GAL(1-15) (Fuxe et al., 2008; Fuxe et al., 2012; Millon et al., 2015; BorrotoEscuela et al., 2014). Therefore, the anhedonia-like effects induced by GAL(1- 
15) are mediated by GALR2, confirming that GAL(1-15) acts through the heterodimer GALR1/GALR2.

Not only in the operant model but also in non-operant models using several reinforcements, such as the natural reinforcer sucrose (SPT) (Willner, 2005; Walser, 2011; Der-Avakian and Markou, 2012), the food (NSF) (Walser, 2011; Ferres-Coy et al., 2016) and the sexual motivation behaviour (FUST) (Malkesman et al., 2010; Malkesman et al., 2012), GAL(1-15) induced a significant anhedonic-effect, confirming an important role for this neuropeptide in anhedonia, which is a core feature of Major Depressive Disorders.

We have used ${ }^{18} \mathrm{~F}-\mathrm{FDG}$ Positron Emission Tomography for in vivo imaging of metabolic processes (Eidelberg, 2009) as a powerful tool to study the effect of GAL(1-15). GAL(1-15) treatment altered glucose metabolism in several nuclei of the limbic system, decreasing FDG uptake in the hippocampus and thalamus or in several areas of the striatum and increasing the uptake in the prefrontal and piriform cortexes. These results are in agreement with previous works in which the hippocampus, the striatum or the prefrontal cortex were targeted for GAL(115) (Millon et al., 2015; Millon et al., 2016; Millon et al., 2017a) (Flores-Burgess, 2018), which are areas rich in GAL-fragment binding sites (Hedlund et al., 1992). However, we cannot disregard GAL(1-15) action in other small nuclei such as the dorsal raphe (Millon et al., 2015), VTA or NAc because of the limited resolution of PET scans (Prieto et al., 2011).

We further investigated GAL(1-15) effects on the key mesolimbic reward nodes, the VTA and the NAc, by evaluating the effects of GAL(1-15) treatment on the expression of genes that regulate dopaminergic activity, which is critical for the 
reward system and anhedonia (Russo and Nestler, 2013; Nestler and Carlezon, 2006; Bressan and Crippa, 2005). GAL(1-15) induced a strong reduction in the expression of the dopamine transporters DAT and Vmat2 in the VTA. Since DAT regulates extracellular DA levels in the extracellular space into presynaptic DA terminals after DA release and since Vmat2 packages monoamines into synaptic vesicles for subsequent release (Garcia-Perez et al., 2016), our results suggest that $\mathrm{GAL}(1-15)$ affects the production and release of DA. In addition, GAL(1-15) also increased D3 receptor expression in the VTA, which is a receptor localized not only in the postsynaptic region but also in the presynaptic region, where it modulates both dopamine synthesis and release, thereby acting as an autoreceptor (Leggio et al., 2013; Diaz et al., 2000).

Moreover, the colocalization, as determined by procedures previously used (Millon et al., 2015), of GALR1 and GALR2 in the VTA (Figure S1A) and the increased GALR1 and GALR2 expression induced by GAL(1-15) in this area confirmed that the VTA is a target of GAL(1-15). Since GAL(1-15) acts through a GALR1/GALR2 heteroreceptor complex that inhibits the Gq/G11-mediated signalling of the GALR2 protomer and switches it towards Gi/o-mediated signalling, thereby enhancing Gi/o-mediated signalling of GALR1 in the GALR1-GALR2 heterodimer (Millon et al., 2015; Borroto-Escuela et al., 2014). This enhancement of the Gio-mediated signal reduces DA formation in this cell, which could explain the functional results found in this work.

In the NAc, GAL(1-15) reduced C-Fos mRNA expression and $\mathrm{TH}$ immunoreactivity and increased the numbers of the D1, D2 and D3 receptors. The reduction of DA release suggested by our experiments would explain the 
reduction in neural activity (Manning et al., 2017) in the NAc and can explain the increase in DA receptor expression as a compensatory effect.

Altogether, these modifications induced by GAL 1-15 suggested that its main effect on the mesolimbic pathway is the structural and functional reorganization of the dopaminergic system, which likely leads to its functional inhibition.

The results of the present work are in agreement with previous work that showed that the effect of GAL(1-15) in EtOH consumption was directly related to the dopaminergic system and the striatum (Millon et al., 2017a).

In conclusion, our results indicate that GAL(1-15) induces a strong anhedonialike phenotype in rats, likely due to the involvement of the dopaminergic mesolimbic system, which is critical for the reward system and anhedonia (Russo and Nestler, 2013; Nestler and Carlezon, 2006; Gold et al., 2018; Bressan and Crippa, 2005). Motivation is a brain function that is based on this dopaminergic system, which is critically compromised in several neuropsychiatric diseases/conditions, including depression. Our results may help to better understand the complex problem of impaired motivation (i.e., anhedonia) and may provide a basis for the development of novel therapeutic strategies using $\mathrm{GAL}(1-15)$ analogues for the treatment of depression and reward-related diseases.

\section{ACKNOWLEDGMENTS AND DISCLOSURES}

We are grateful to the Unidad de Imagen Molecular of Centro de Investigación Medico-Sanitaria (CIMES) from Universidad de Málaga for their valuable support in the PET experiments. This study was supported by grants awarded by the Spanish Ministry of Economy (SAF2016-79008-P) and (PSI2017-82604- 
R) and by the University of Málaga (Proyecto Jóvenes Investigadores PPIT.

UMA.B1.2017/17). C. M. was supported by a fellowship from the University of Málaga (Contrato Postoctoral UMA).

The authors report no biomedical financial interests or potential conflicts of interest.

\section{REFERENCES}

Albert PR, Le Francois B and Millar AM. (2011) Transcriptional dysregulation of 5HT1A autoreceptors in mental illness. Mol Brain 4: 21.

Alen F, Santos A, Moreno-Sanz G, et al. (2009) Cannabinoid-induced increase in relapse-like drinking is prevented by the blockade of the glycine-binding site of N-methyl-D-aspartate receptors. Neuroscience 158(2): 465-473.

Barde S, Ruegg J, Prud'homme J, et al. (2016) Alterations in the neuropeptide galanin system in major depressive disorder involve levels of transcripts, methylation, and peptide. Proc Natl Acad Sci U S A 113(52): E8472-E8481.

Barr AM, Kinney JW, Hill MN, et al. (2006) A novel, systemically active, selective galanin receptor type-3 ligand exhibits antidepressant-like activity in preclinical tests. Neurosci Lett 405(1-2): 111-115.

Bartfai T, Lu X, Badie-Mahdavi H, et al. (2004) Galmic, a nonpeptide galanin receptor agonist, affects behaviors in seizure, pain, and forced-swim tests. Proc Natl Acad Sci U S A 101(28): 10470-10475.

Bellido I, Diaz-Cabiale Z, Jimenez-Vasquez PA, et al. (2002) Increased density of galanin binding sites in the dorsal raphe in a genetic rat model of depression. Neurosci Lett 317(2): 101-105.

Borroto-Escuela DO, Narvaez M, Di Palma M, et al. (2014) Preferential activation by galanin 1-15 fragment of the GalR1 protomer of a GalR1-GalR2 heteroreceptor complex. Biochem Biophys Res Commun.

Branchek TA, Smith KE, Gerald C, et al. (2000) Galanin receptor subtypes. Trends Pharmacol Sci 21(3): 109-117.

Bressan RA and Crippa JA. (2005) The role of dopamine in reward and pleasure behaviour--review of data from preclinical research. Acta Psychiatr Scand Suppl (427): 14-21.

Cadoni C, Pisanu A, Simola N, et al. (2017) Widespread reduction of dopamine cell bodies and terminals in adult rats exposed to a low dose regimen of MDMA during adolescence. Neuropharmacology 123: 385-394.

Der-Avakian A and Markou A. (2012) The neurobiology of anhedonia and other reward-related deficits. Trends Neurosci 35(1): 68-77.

Diaz J, Pilon C, Le Foll B, et al. (2000) Dopamine D3 receptors expressed by all mesencephalic dopamine neurons. J Neurosci 20(23): 8677-8684.

Diaz-Cabiale Z, Parrado C, Fuxe K, et al. (2007) Receptor-receptor interactions in central cardiovascular regulation. Focus on neuropeptide/alpha(2)adrenoreceptor interactions in the nucleus tractus solitarius. J Neural Transm 114(1): 115-125. 
Diaz-Cabiale Z, Parrado C, Narvaez M, et al. (2010) Neurochemical modulation of central cardiovascular control: the integrative role of galanin. EXS 102: 113131.

Diaz-Cabiale Z, Parrado C, Vela C, et al. (2005) Role of galanin and galanin(1-15) on central cardiovascular control. Neuropeptides 39(3): 185-190.

Eidelberg D. (2009) Metabolic brain networks in neurodegenerative disorders: a functional imaging approach. Trends Neurosci 32(10): 548-557.

Ettenberg A. (2009) The runway model of drug self-administration. Pharmacol Biochem Behav 91(3): 271-277.

Ferres-Coy A, Galofre M, Pilar-Cuellar F, et al. (2016) Therapeutic antidepressant potential of a conjugated siRNA silencing the serotonin transporter after intranasal administration. Mol Psychiatry 21(3): 328-338.

Flores-Burgess A, Millon C, Gago B, et al. (2017) Galanin (1-15) enhancement of the behavioral effects of Fluoxetine in the forced swimming test gives a new therapeutic strategy against depression. Neuropharmacology 118: 233-241.

Flores-Burgess A, Millon, C., Gago, B., García-Durán, L., Cantero-García, N., Coveñas, R., Naváez, JA., Fuxe, K., Santín, L., Díaz-Cabiale, Z. . (2018) GALANIN(1-15)FLUOXETINE INTERACTIONS IN THE PREFRONTAL CORTEX: INVOLVEMENT OF GALR1-GALR2-5-HT1A HETERORECEPTOR COMPLEXES. Neuropharmacology (Submitted).

Fuxe K, Borroto-Escuela DO, Romero-Fernandez W, et al. (2012) On the existence and function of galanin receptor heteromers in the central nervous system. Front Endocrinol (Lausanne) 3: 127.

Fuxe K, Marcellino D, Rivera A, et al. (2008) Receptor-receptor interactions within receptor mosaics. Impact on neuropsychopharmacology. Brain Res Rev 58(2): 415-452.

Garcia-Perez D, Nunez C, Laorden ML, et al. (2016) Regulation of dopaminergic markers expression in response to acute and chronic morphine and to morphine withdrawal. Addict Biol 21(2): 374-386.

Genders SG, Scheller KJ and Djouma E. (2018) Neuropeptide modulation of addiction: Focus on galanin. Neurosci Biobehav Rev.

Gold MS, Blum K, Febo M, et al. (2018) Molecular role of dopamine in anhedonia linked to reward deficiency syndrome (RDS) and anti- reward systems. Front Biosci (Schol Ed) 10: 309-325.

Gorwood P. (2008) Neurobiological mechanisms of anhedonia. Dialogues Clin Neurosci 10(3): 291-299.

Hedlund PB and Fuxe K. (1996) Galanin and 5-HT1A receptor interactions as an integrative mechanism in 5-HT neurotransmission in the brain. Ann NY Acad Sci 780: 193-212.

Hedlund PB, Yanaihara N and Fuxe K. (1992) Evidence for specific N-terminal galanin fragment binding sites in the rat brain. Eur J Pharmacol 224(2-3): 203-205.

Jacobowitz DM, Kresse A and Skofitsch G. (2004) Galanin in the brain: chemoarchitectonics and brain cartography--a historical review. Peptides 25(3): 433-464.

Juhasz G, Hullam G, Eszlari N, et al. (2014) Brain galanin system genes interact with life stresses in depression-related phenotypes. Proc Natl Acad Sci U S A 111(16): E1666-1673. 
Kormos V and Gaszner B. (2013) Role of neuropeptides in anxiety, stress, and depression: from animals to humans. Neuropeptides 47(6): 401-419.

Kuteeva E, Hokfelt $T$ and Ogren SO. (2005) Behavioural characterisation of transgenic mice overexpressing galanin under the PDGF-B promoter. Neuropeptides 39(3): 299-304.

Kuteeva E, Hokfelt T, Wardi T, et al. (2008a) Galanin, galanin receptor subtypes and depression-like behaviour. Cell Mol Life Sci 65(12): 1854-1863.

Kuteeva E, Wardi T, Hokfelt T, et al. (2007) Galanin enhances and a galanin antagonist attenuates depression-like behaviour in the rat. Eur Neuropsychopharmacol 17(1): 64-69.

Kuteeva E, Wardi T, Lundstrom L, et al. (2008b) Differential role of galanin receptors in the regulation of depression-like behavior and monoamine/stress-related genes at the cell body level. Neuropsychopharmacology 33(11): 2573-2585.

Leggio GM, Salomone S, Bucolo C, et al. (2013) Dopamine D(3) receptor as a new pharmacological target for the treatment of depression. Eur J Pharmacol 719(1-3): 25-33.

Lu X, Barr AM, Kinney JW, et al. (2005) A role for galanin in antidepressant actions with a focus on the dorsal raphe nucleus. Proc Natl Acad Sci U S A 102(3): 874-879.

Malkesman O, Austin DR, Tragon T, et al. (2012) Acute D-serine treatment produces antidepressant-like effects in rodents. Int J Neuropsychopharmacol 15(8): 1135-1148.

Malkesman O, Scattoni ML, Paredes D, et al. (2010) The female urine sniffing test: a novel approach for assessing reward-seeking behavior in rodents. Biol Psychiatry 67(9): 864-871.

Manning CE, Williams ES and Robison AJ. (2017) Reward Network Immediate Early Gene Expression in Mood Disorders. Front Behav Neurosci 11: 77.

Marco EM, Ballesta JA, Irala C, et al. (2017) Sex-dependent influence of chronic mild stress (CMS) on voluntary alcohol consumption; study of neurobiological consequences. Pharmacol Biochem Behav 152: 68-80.

Marquez PV and Saxena S. (2016) Making Mental Health a Global Priority. Cerebrum 2016.

Millon C, Flores-Burgess A, Castilla-Ortega E, et al. (2017a) Central administration of galanin N-terminal fragment 1-15 decreases the voluntary alcohol intake in rats. Addict Biol.

Millon C, Flores-Burgess A, Narvaez M, et al. (2017b) The neuropeptides Galanin and Galanin(1-15) in depression-like behaviours. Neuropeptides 64: 39-45.

Millon C, Flores-Burgess A, Narvaez M, et al. (2016) Galanin (1-15) enhances the antidepressant effects of the 5-HT1A receptor agonist 8-OH-DPAT: involvement of the raphe-hippocampal 5-HT neuron system. Brain Struct Funct 221(9): 4491-4504.

Millon C, Flores-Burgess A, Narvaez M, et al. (2015) A role for galanin N-terminal fragment (1-15) in anxiety- and depression-related behaviors in rats. Int J Neuropsychopharmacol 18(3): 1-13.

Mitsukawa K, Lu X and Bartfai T. (2008) Galanin, galanin receptors and drug targets. Cell Mol Life Sci 65(12): 1796-1805.

Nestler EJ and Carlezon WA, Jr. (2006) The mesolimbic dopamine reward circuit in depression. Biol Psychiatry 59(12): 1151-1159. 
Pascual-Brazo J, Castro E, Diaz A, et al. (2012) Modulation of neuroplasticity pathways and antidepressant-like behavioural responses following the short-term (3 and 7 days) administration of the 5-HT(4) receptor agonist RS67333. Int J Neuropsychopharmacol 15(5): 631-643.

Prieto E, Collantes M, Delgado M, et al. (2011) Statistical parametric maps of (1)(8)F-FDG PET and 3-D autoradiography in the rat brain: a crossvalidation study. Eur J Nucl Med Mol Imaging 38(12): 2228-2237.

Russo SJ and Nestler EJ. (2013) The brain reward circuitry in mood disorders. Nat Rev Neurosci 14(9): 609-625.

Sanchis-Segura C and Spanagel R. (2006) Behavioural assessment of drug reinforcement and addictive features in rodents: an overview. Addict Biol 11(1): 2-38.

Swanson CJ, Blackburn TP, Zhang X, et al. (2005) Anxiolytic- and antidepressantlike profiles of the galanin-3 receptor (Gal3) antagonists SNAP 37889 and SNAP 398299. Proc Natl Acad Sci U S A 102(48): 17489-17494.

Tatemoto K, Rokaeus A, Jornvall H, et al. (1983) Galanin - a novel biologically active peptide from porcine intestine. FEBS Lett 164(1): 124-128.

Walser SMaDJM. (2011) Mouse Models of Depression. In: Uehara DT (ed) Psychiatric Disorders-Trends and Developments. In Tech.

Weiss JM, Bonsall RW, Demetrikopoulos MK, et al. (1998) Galanin: a significant role in depression? Ann N Y Acad Sci 863: 364-382.

Willner P. (2005) Chronic mild stress (CMS) revisited: consistency and behavioural-neurobiological concordance in the effects of CMS. Neuropsychobiology 52(2): 90-110. 
Figure 1. Experimental design of the procedures for saccharin selfadministration (A), the sucrose preference test (SPT) (B), and the novelty suppressed feeding test (NSF) and female urine sniffing test (C). i.c.v, intracerebroventricular.

Figure 2. Role of Galanin (1-15) [GAL(1-15)] on operant self-administration of saccharin $0,2 \%$ in rats. A) Dose-response curve of the effect $G A L(1-15)$ on selfadministration of saccharin. GAL(1-15) ( $n=7-10$ animals) was administered intracerebroventricularly (i.c.v.) $15 \mathrm{~min}$ before the test session. Cerebrospinal fluid-injected rats were used as the control group ( $n=12$ animals). B) The effect of administration of Galanin (GAL) and GAL(1-15) on the self-administration of saccharin. GAL (3 nmol/rat) and GAL(1-15) (3 nmol/rat; $n=8-9$ animals) were injected i.c.v. 15 min before the test. Cerebrospinal fluid-injected rats were used as the Control group ( $n=14$ animals). C) The effects of the co-administration of GALR2 receptor antagonist M871 (3 nmol/rat) and GAL(1-15) ( $\mathrm{n}=7-9$ animals) on the self-administration of saccharin. Treatments were injected i.c.v. $15 \mathrm{~min}$ before the test. Cerebrospinal fluid-injected rats were used as Control groups ( $n=9$ animals). Vertical bars represent a mean \pm standard error of the mean of the Number of saccharin Reinforcements during the test period. ${ }^{*} p<0.05$ and ${ }^{* *} p<0.01$ versus the rest of the groups according to a one-way ANOVA followed by Fisher's least significance difference test.

Figure 3. Effect of administration of Galanin (1-15) [GAL(1-15)] in the sucrose preference test with $1 \%$ sucrose concentration in rats. GAL(1-15) $(3 \mathrm{nmol} / \mathrm{rats}$; $n=8-9$ animals per group) were administered intracerebroventricularly 2, 8 and $24 \mathrm{~h}$ before the measures. Cerebrospinal fluid-injected rats were used as the Control group ( $n=7$ animals). Vertical bars represent the mean \pm standard error of the mean of sucrose intake $(\mathrm{g} / \mathrm{kg})(A$,$) and preference (percent) (B) during$ the different period. ${ }^{*} p<0.05$ and ${ }^{* *} p<0.01$ versus the Control group according to a repeated measures two-way ANOVA followed by Fisher's least significance difference test.

Figure 4. Effect of the administration of Galanin (1-15) [GAL(1-15)] in the novelty-suppressed feeding test (NSF) (A) and the female urine sniffing test (FUST) (B,C) in rats. (A) GAL(1-15) ( $3 \mathrm{nmol} / \mathrm{rat}$; $\mathrm{n}=9$ animals per group) were administered intracerebroventricularly $15 \mathrm{~min}$ before the test. Cerebrospinal fluid-injected rats were used as the Control group ( $n=8$ animals). Vertical bars represent the mean \pm standard error of the mean of the latency period of the first feeding (s) during the test. ${ }^{* * *} \mathrm{p}<0.001$ versus Control group according to a Student's t-test. (B) GAL(1-15) (3 nmol/rat; $n=7$ animals) were administered i.c.v. $15 \mathrm{~min}$ before the test. Cerebrospinal fluid-injected rats (CSFa) were used as the Urine-CSFa group ( $n=6$ animals). Vertical bars represent the mean \pm standard error of the mean of the sniffing duration of water $(n=14$ animals) or oestrus female urine (s) during the test. ${ }^{* * *} p<0.001 ;{ }^{* *} p<0.01$ and ${ }^{*} p<0.05$ according to a one-way ANOVA followed by Fisher's least significant difference test. (C) $\mathrm{GAL}(1-15)$ ( $3 \mathrm{nmol} / \mathrm{rat} ; \mathrm{n}=6$ animals) were administrated icv $15 \mathrm{~min}$ before the test. Cerebrospinal fluid-injected rats (CSFa) were used as the Water-CSFa group ( $n=6$ animals). Data represent the mean \pm standard error of the mean of sniffing duration of water during the test. No differences were found according Student's t-test. 
Figure 5. Effects of GAL(1-15) on rat brain glucose metabolism. Statistical parametric mapping (SPM) analysis results displayed on coronal, horizontal and sagittal brain sections showing regions with significantly reduced (hypometabolism, blue) and increased (hypermetabolism, red) uptake of $\left[{ }^{18} \mathrm{~F}\right] \mathrm{FDG}$ at 30,60 and $90 \mathrm{~min}$ after i.c.v. GAL(1-15) administration $(p<0.01$ uncorrected, clusters with extended threshold $\mathrm{k}=100$ ).

Figure 6. Effects of Galanin (1-15) [GAL(1-15)] in the ventral tegmental area (VTA) mRNA expression of (A) Dat, (B) Vmat2, (C) C-Fos or nucleus accumbens (NAc) mRNA expression of (D) C-Fos in rats. GAL(1-15) (3 $\mathrm{nmol} / \mathrm{rat}$ ) was injected intracerebroventricularly 1 hour before the measures. Cerebrospinal fluid-injected rats were used as the Control group. Vertical bars represent the mean \pm standard error of the mean ( $n=4-5$ animals per group). ${ }^{*} p<0.05$ versus Control group according to a Student's $t$-test.

Figure 7. Effects of Galanin (1-15) [GAL(1-15)] in the expression of the dopamine receptors $D 1, D 2, D 3$ and $D 5$ in the ventral tegmental area (VTA) (AD) or nucleus accumbens (NAc) (E-H) in rats. GAL(1-15) (3 nmol/rat) was injected intracerebroventricularly 1 hour before the measures. Cerebrospinal fluid-injected rats were used as the Control group. Vertical bars represent the mean \pm standard error of the mean ( $n=4-5$ animals per group). ${ }^{*} p<0.05$ versus Control group according to a Student's $t$-test. 
Millón et al., 2018 Journal of Psychopharmacology

Table 1. Galr1 and Galr2 mRNA expression.

Galr1

Galr2

Control GAL(1-15) Control

GAL(1-15)

VTA

$0.70 \pm 0.2$

$1.21 \pm 0.4$

$1.22 \pm 0.1$

$1.66 \pm 0.1^{*}$

NAC

$0.72 \pm 0.2$

$0.47 \pm 0.1$

$0.99 \pm 0.1$

$0.98 \pm 0.1$

Table 1. Effects of Galanin (1-15) [GAL(1-15)] in the expression of Galanin receptors Galr1 and Galr2 in the Ventral Tegmental Area (VTA) or Nucleus Accubens (NAc) in rats. GAL(1-15) (3nmol/rat) was injected intracerebroventricular 1 hour before the measures. Cerebrospinal fluid-injected rats were used as Control group. $n=4-5$ animals per group. ${ }^{*} p<0.05$ versus Control group according to Student's $t$-test.

Table 2. Tyrosine Hydroxylase immunoreactivity in the Striatum.

\begin{tabular}{ccc}
\hline & Control & GAL(1-15) \\
\hline NAc Shell & $24.2 \pm 4.7$ & $16.5 \pm 4.0$ \\
NAc Core & $6.7 \pm 0.3$ & $4.5 \pm 0.7^{*}$ \\
\hline Dorsal Striatum (CPu) & & $4.9 \pm 0.8$ \\
\hline
\end{tabular}


A.
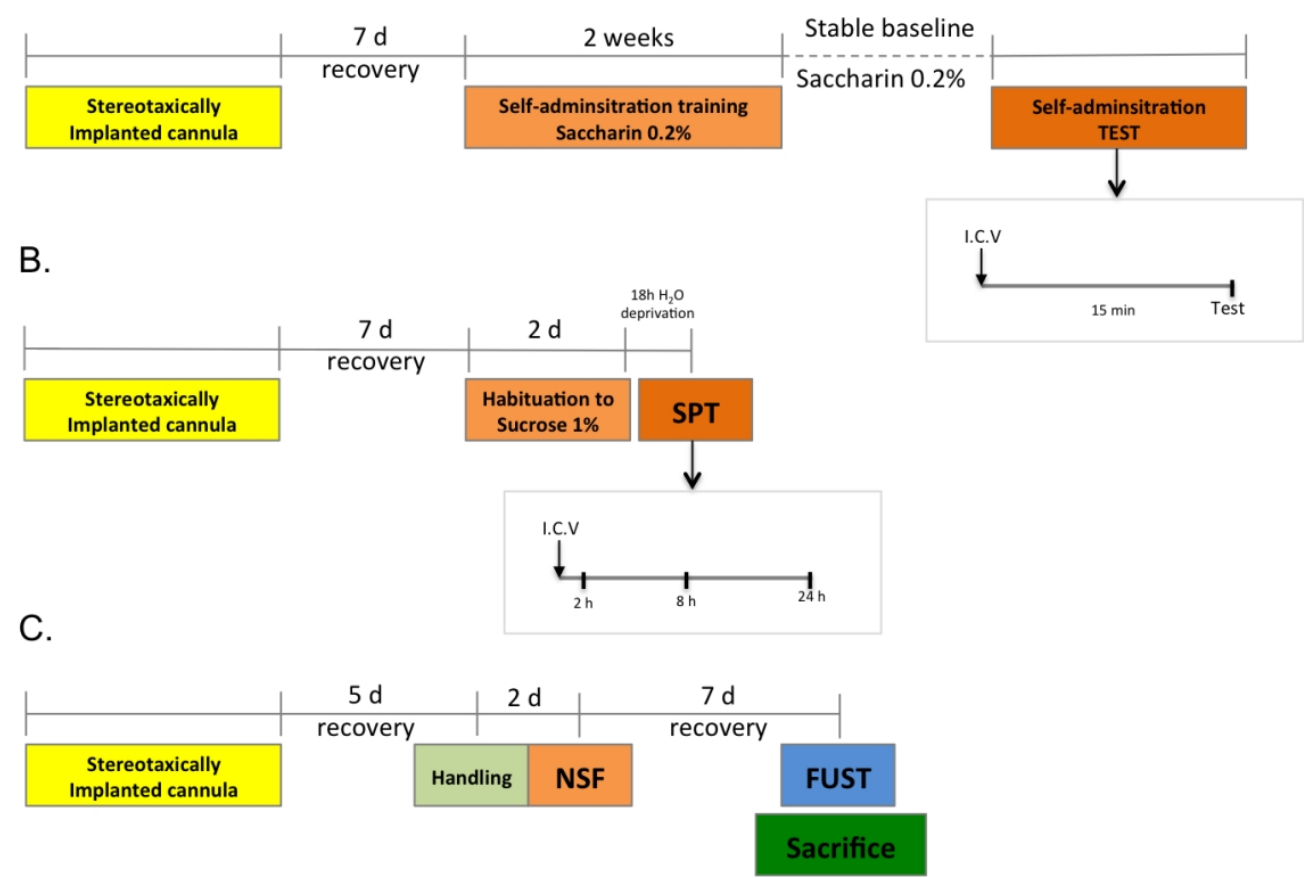

Figure 1

$231 \times 168 \mathrm{~mm}(150 \times 150 \mathrm{DPI})$

http://mc.manuscriptcentral.com/jop 
A.

B.

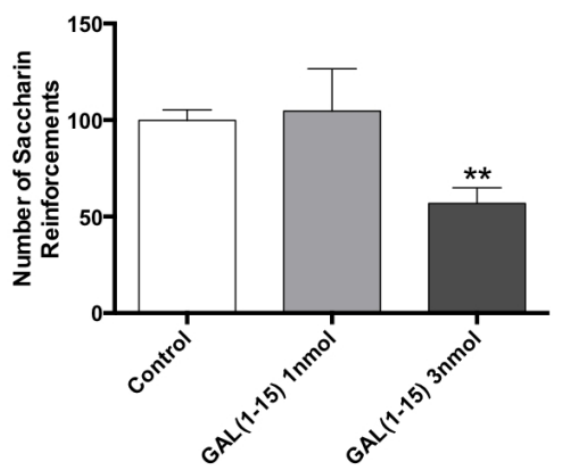

C.
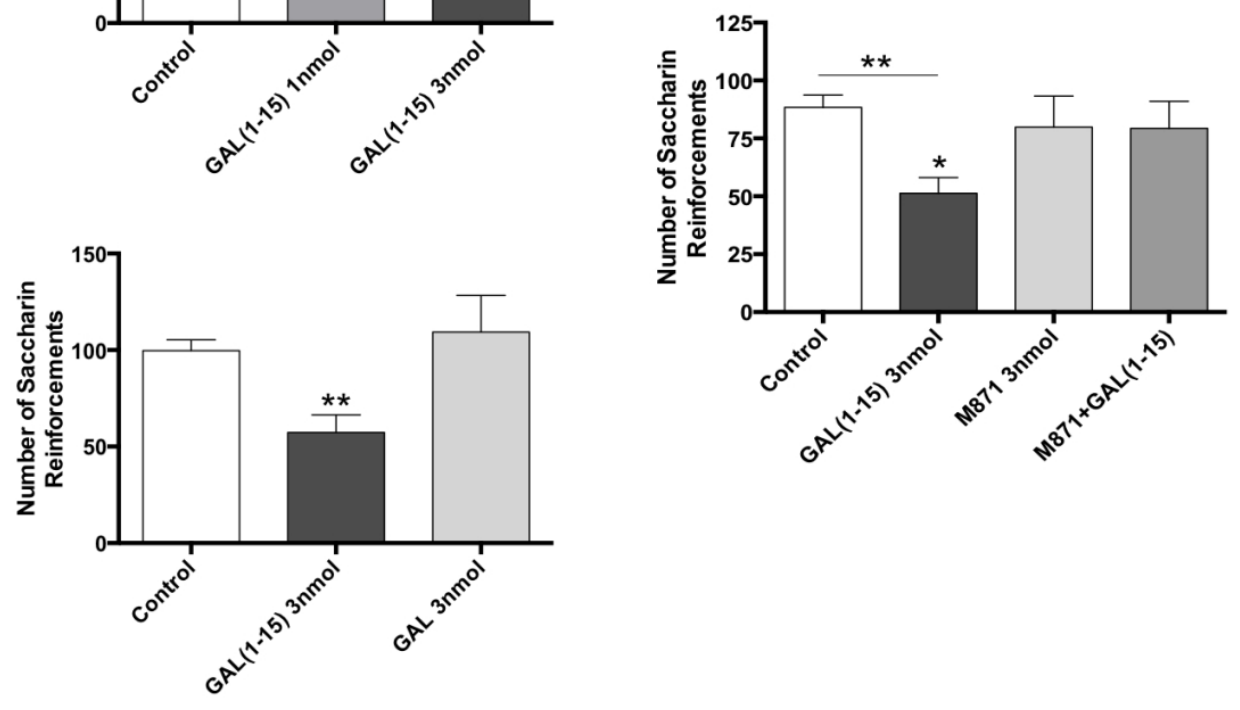

Figure 2

$227 \times 185 \mathrm{~mm}(150 \times 150 \mathrm{DPI})$

http://mc.manuscriptcentral.com/jop 

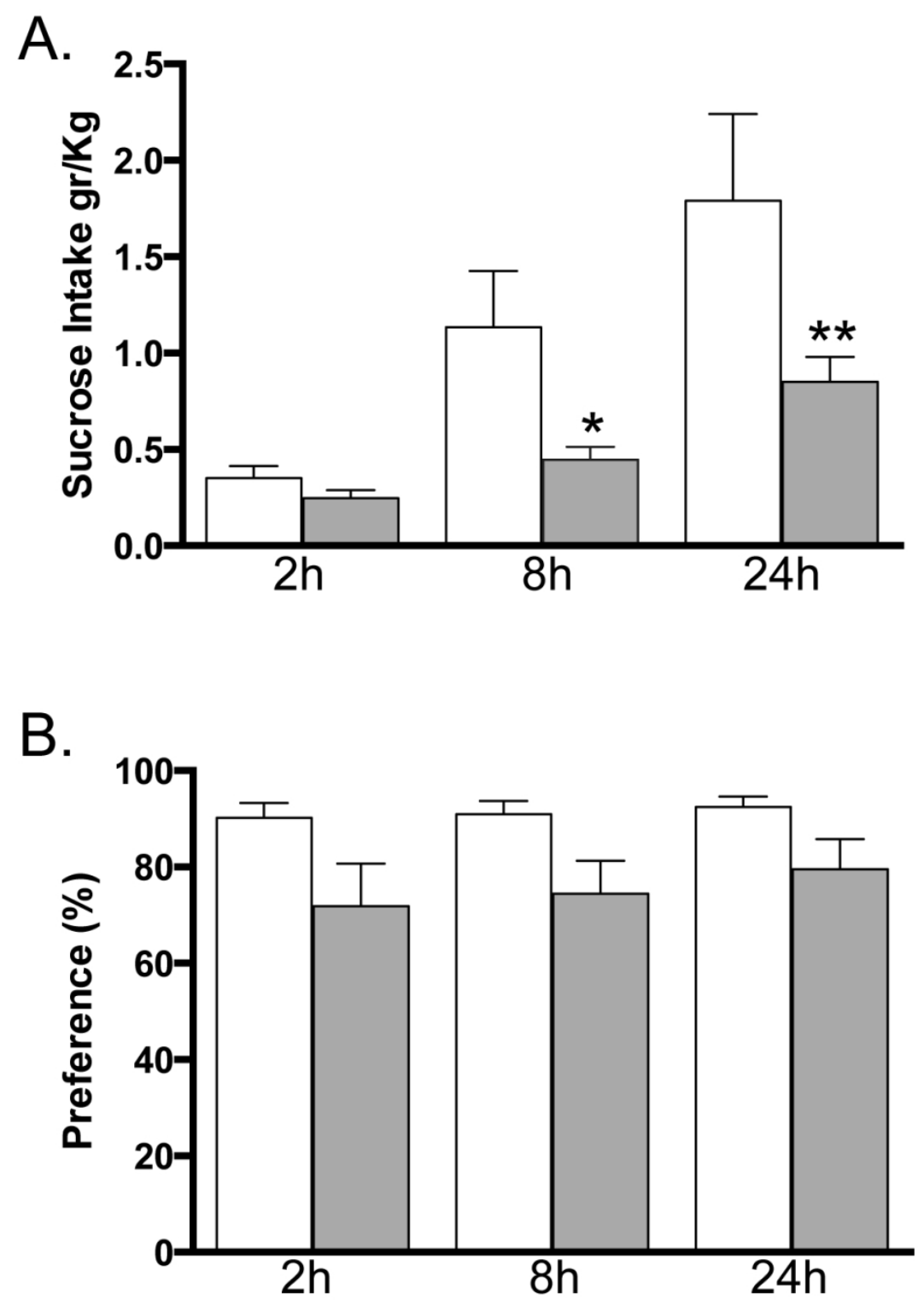

Figure 3 revised

$99 \times 140 \mathrm{~mm}(300 \times 300 \mathrm{DPI})$ 


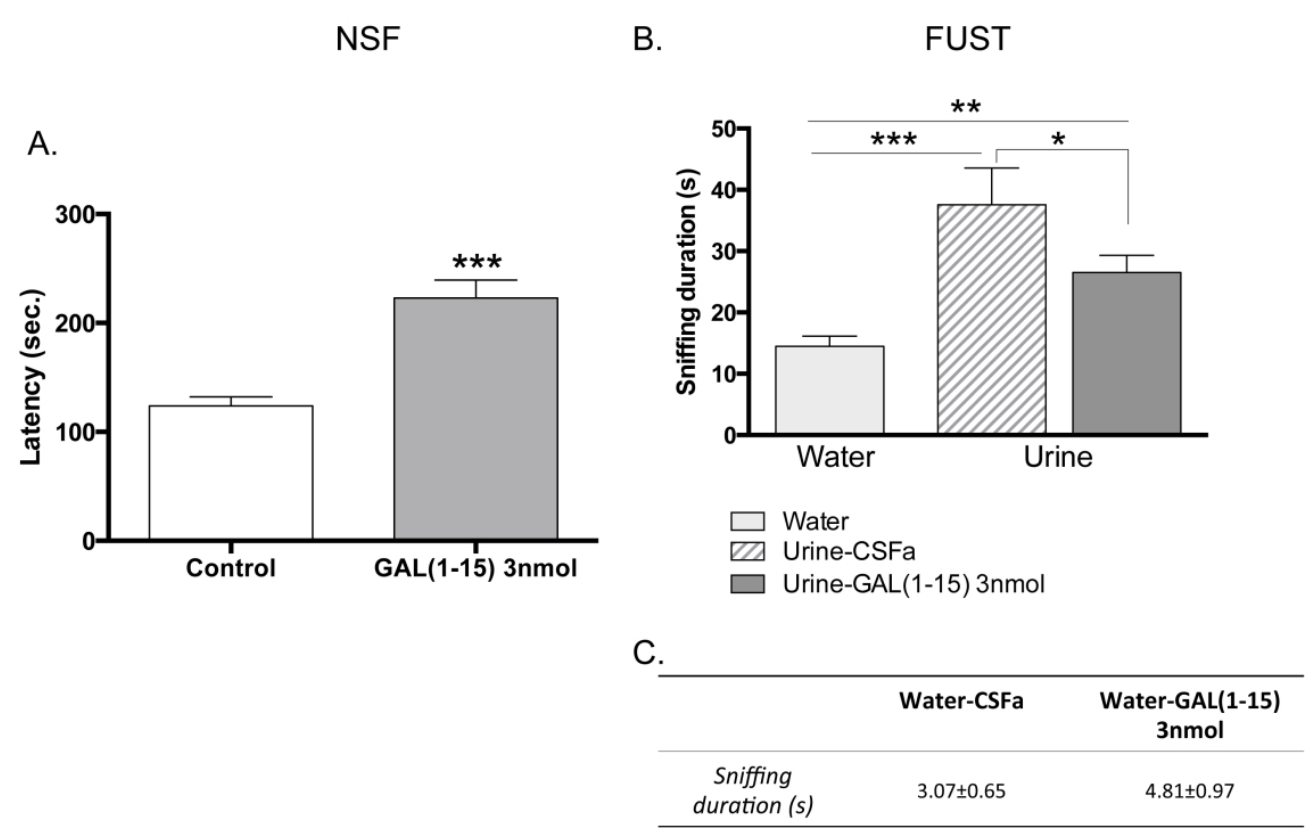

Figure 4 revised

$249 \times 156 \mathrm{~mm}(300 \times 300$ DPI) 


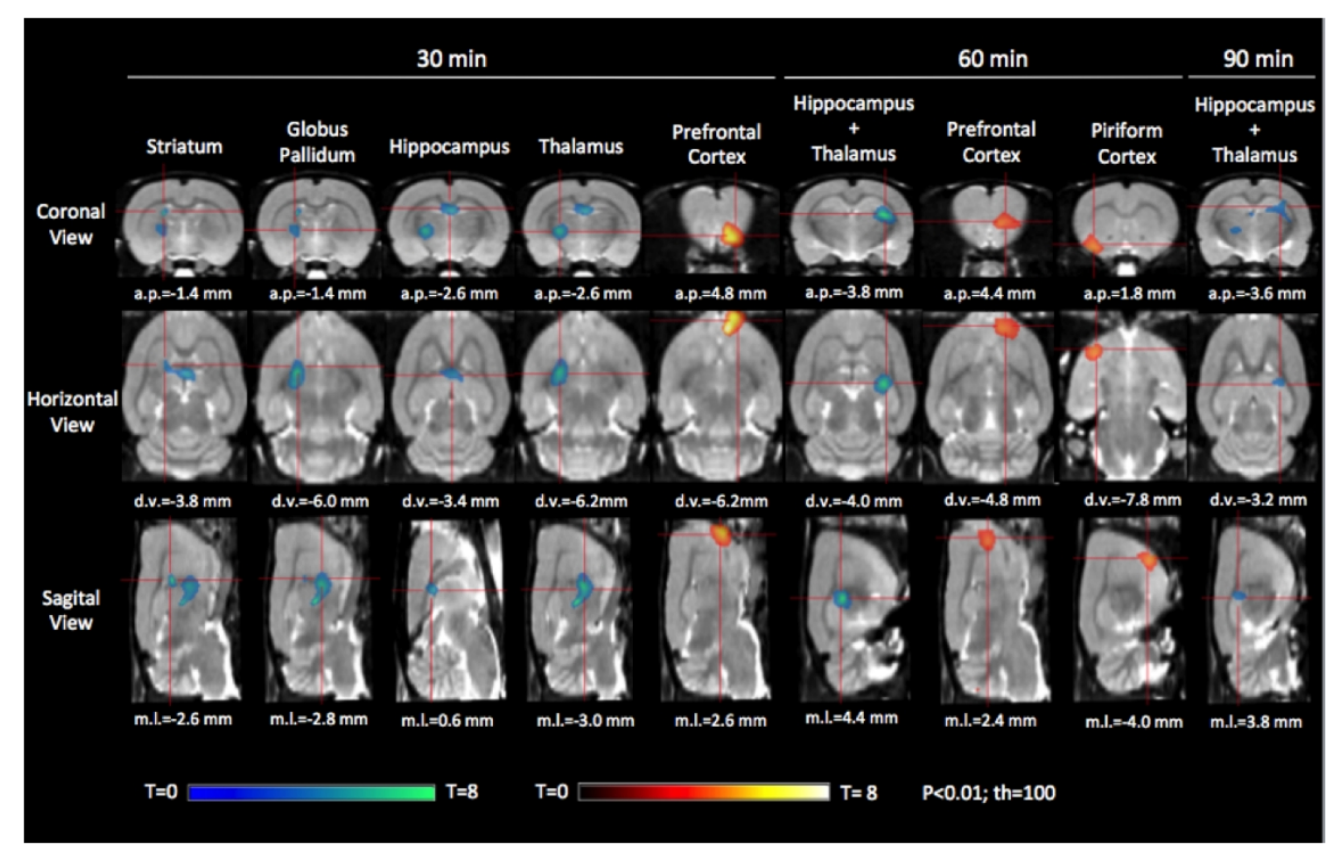

Figure 5

$225 \times 143 \mathrm{~mm}(150 \times 150 \mathrm{DPI})$ 

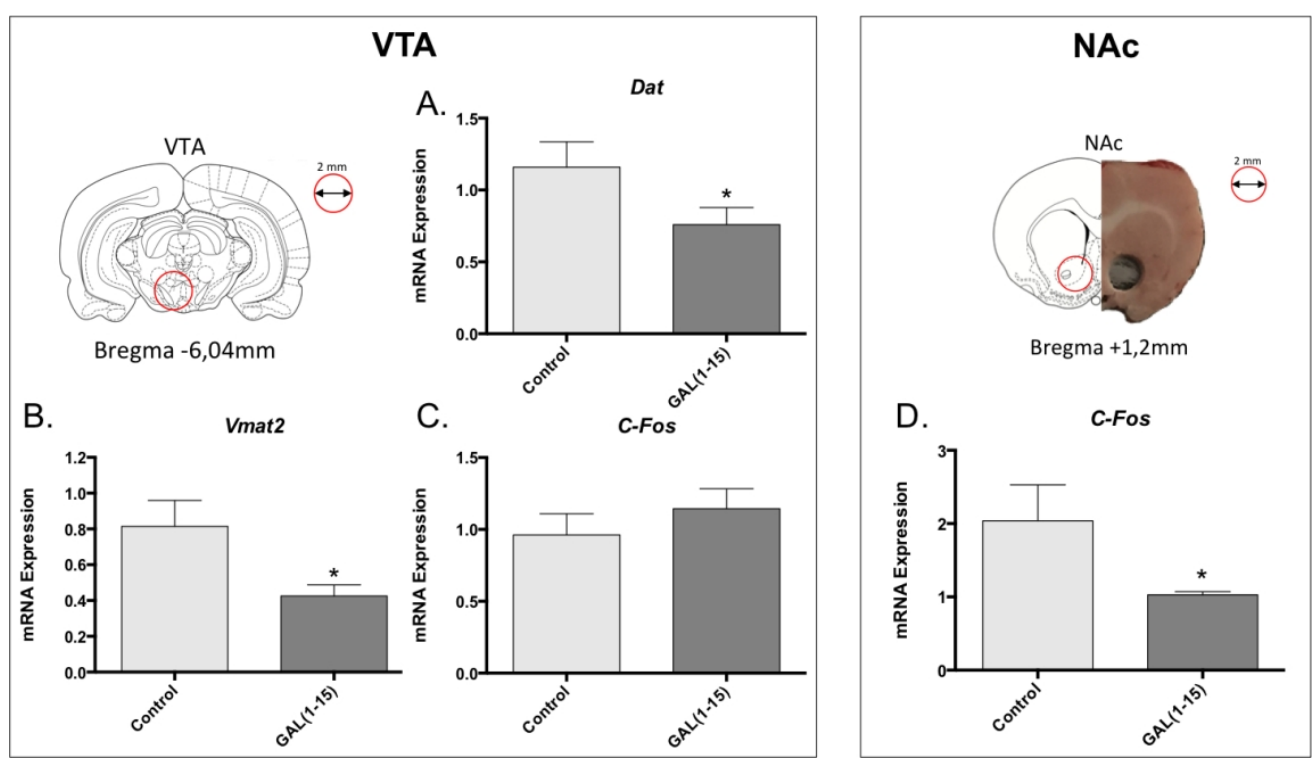

Figure 6

$245 \times 139 \mathrm{~mm}(150 \times 150 \mathrm{DPI})$ 

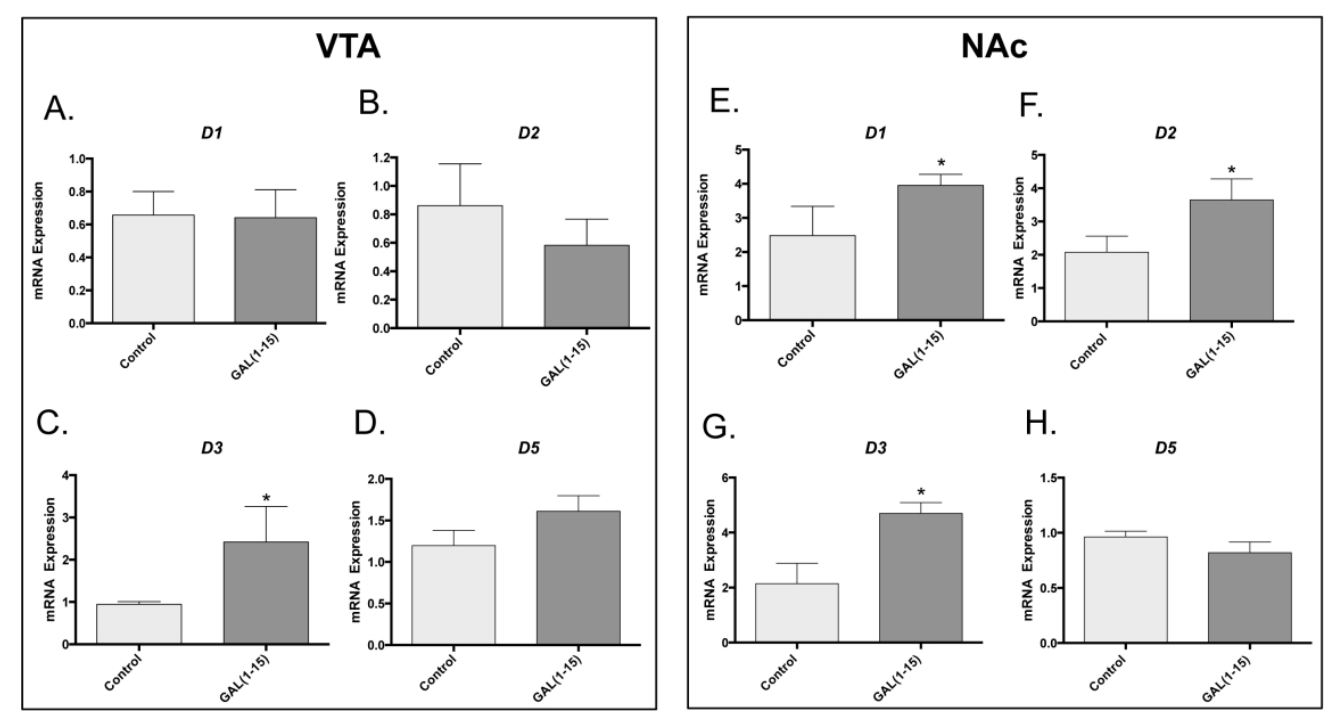

Figura 7 revised

$244 \times 130 \mathrm{~mm}(300 \times 300$ DPI) 


\section{SUPPLEMENTARY MATERIAL AND METHODS}

\section{Animals}

Male Sprague Dawley rats (body weight 225-250g, age 8 weeks) were obtained from Criffa and maintained in a humidity- and temperature-controlled $\left(20-22^{\circ} \mathrm{C}\right)$ room. The rats in the saccharin self-administration and sucrose preference test were during the entire protocol maintained on a 12-hour reversed light/dark cycle (lights off at $10 \mathrm{am}$ ) whereas the other rats kept on 12/hour light/dark cycle. The animals had free access to food pellets and tap water. Experimental procedures were approved by the Institutional Animal Ethics Committee of the University of Málaga, in accordance with the European Directive (86/609/EEC) and Spanish Directive (Real Decretory 53/2013).

\section{Intracerebroventricular Injections}

This protocol has been used previously (Millon et al., 2015; Diaz-Cabiale et al., 2011). Briefly, the rats were anesthetized intraperitoneally with equitesin $(3.3 \mathrm{~mL} / \mathrm{kg}$ body weight), and stereotaxically implanted with a unilateral chronic 22-gauge stainless-steel guide cannula into the right lateral cerebral ventricle using the following coordinates: $1.4 \mathrm{~mm}$ lateral and $1 \mathrm{~mm}$ posterior to bregma, and $3.6 \mathrm{~mm}$ below the surface of the skull (Paxinos, 1986). After surgery, animals were individually housed and allowed a recovery period of 7 days. The injections in the lateral ventricle were performed using a 26-gauge stain lesssteel injection cannula connected via a PE-10 tubing to a Hamilton syringe. The total volume was $5 \mu \mathrm{l}$ per injection and the infusion time was $1 \mathrm{~min}$.

Solutions were prepared freshly and the peptides were dissolved in artificial cerebrospinal fluid (composition is $120 \mathrm{nM} \mathrm{NaCl}, 20 \mathrm{nM} \mathrm{NaH} \mathrm{N}_{2} \mathrm{CO}_{3}, 2 \mathrm{nM} \mathrm{KCl}$,

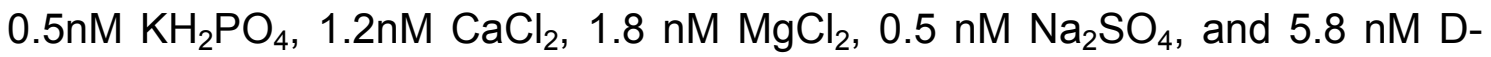
glucose, $\mathrm{pH}$ 7.4). GAL was obtained from NeoMPS, Strasbourg, France; GAL(1-15) and the GALR2 receptor antagonist M871 were obtained from Tocris Bioscience,Bristol,UK. 


\section{Saccharin Self-administration}

Training and testing were conducted in standard operant chambers. Each chamber was equipped with a drinking reservoir $(0.10 \mathrm{ml})$ positioned $4 \mathrm{~cm}$ above the grid floor in the center of the front panel of the chamber, and two retractable levers were located $3 \mathrm{~cm}$ to right and left of the dinking receptacle (one being the active lever, and the other being the inactive lever). Visual stimuli were presented via a light located on the front panel.

\section{Sucrose Preference Test}

Briefly, rats were habituated to $1 \%$ sucrose solution for two days with free access to water and sucrose in their home cage. To prevent possible effects of side preference in drinking behaviour, the position of the bottles was switched every $24 \mathrm{~h}$. After this adaptation, rats were deprived of water for $18 \mathrm{~h}$. Food was available during the testing session.

\section{Novelty Suppressed feeding}

Novelty Suppressed feeding (NSF) is a conflict test that elicits competing motivations: the drive to eat and the fear of venturing into the center of the arena (Ferres-Coy et al., 2016). The testing apparatus consisted of a plastic box $(60 \times 40 \times 20 \mathrm{~cm})$, with its floor covered with wooden bedding $(2 \mathrm{~cm})$ and with a single pellet of food in the center of the box on white circular filter paper $(10 \mathrm{~cm}$ in diameter). Each rat was placed in the corner of the testing arena and the latency of the first feeding episode was recorded. Non-feeding behaviours (e.g. smelling and touching) were ignored.

\section{Female Urine Sniffing Test}

The female Urine Sniffing Test (FUST) is a non-operant test for measuring reward-seeking behaviour in rodents based on interest in sniffing pheromones odours from opposite sex, and was conducted as described previously (Malkesman et al., 2012). Briefly, the test had three phases: (1) a single exposure (3 $\mathrm{min}$ ) to the cotton tip dipped in sterile water, during which the time spent sniffing the applicator was measured; 2 an interval of 45 min during which no applicator was presented to the animal; and (3) a single exposure ( $3 \mathrm{~min}$ ) to 
a cotton tip infused with fresh urine collected from females of the same strain in oestrus or water, during which the time spent sniffing was measured.

The animals receiving GAL(1-15) 3nmol or vehicle were injected 15 min before to the exposure to the urine or water (phase 3).

\section{PET Studies}

Ten or eleven days after surgery, the animals $(n=8 ; 335 \pm 38 \mathrm{~g})$ were fasted overnight and anaesthetized with isoflurane inhalation (5\% for induction and $2 \%$ for maintenance in $100 \%$ oxygen gas). Animals were administered icv with aCSF and after 3-4 days with Gal(1-15) (3 nmol). Immediately thereafter the treatment, the ${ }^{18} \mathrm{~F}-\mathrm{FDG}(43.6 \pm 5.5 \mathrm{MBq}$ in $0.3 \mathrm{ml}$ of $0.9 \% \mathrm{NaCl})$ was injected through the tail vein. After an awake uptake period of 25 minutes to ensure the incorporation of ${ }^{18} \mathrm{~F}-\mathrm{FDG}$ during drug-induce behavioural effects (Prieto et al., 2011), animals were anesthetized and placed prone on the PET scanner bed to perform a static acquisition of $30 \mathrm{~min}$ at three different time-points (30,60 and 90 min) after tracer administration. The images were subsequently reconstructed using an iterative 3-D row action maximum likelihood algorithm (3-D RAMLA), with 2 iterations and a relaxation parameter of 0.024 . Corrections for dead time, decay and random coincidences were applied. Images were reconstructed on a $128 \times 128 \times 120$ matrix, where the voxel size equals $1 \times 1 \times$ $1 \mathrm{~mm}$.

Blood glucose concentration (128 $\pm 24 \mathrm{mg} / \mathrm{dl})$ was determine before tracer injection, using a glucose level meter with test strips (Accu Chek Aviva Nano, Roche, Mannhelm, Germany).

Image preprocessing

The image preprocessing was performed using PMOD (PMOD Technologies, Zürich, Switzerland; https://www.pmod.com/web/), SPM8 (Wellcome Department of Imaging Neuroscience, London, UK; http://www.fil.ion.ucl.ac.uk/spm/software/spm8/), SAMIT (Small Animal Molecular Imaging Toolbox created by University Medical Center Groningen for SPM, Groningen, Netherlands; http://mic-umcg.github.io/samit/) and a custommade software develop using MATLAB R2015b (Mathworks; 
https://es.mathworks.com/products/new_products/release2015b.html)

to normalize the ${ }^{18} \mathrm{~F}-\mathrm{FDG}$ uptake values to a whole brain average value.

The PET images were initially manually matched using PMOD Fusion toolbox to a T2-MRI rat template of SAMIT (Technologies, 2016), which is in Paxinos and Watson anatomical space (Schwarz et al., 2006; (UMCG). 2015). This coregister included several rotations and translations in each of the three dimensions ( $x, y$ and $z$ ) to match the location, and orientation of the T2 template. Afterwards, the coregistered images were spatially normalized to a FDG rat template provided by SAMIT (Vallez Garcia et al., 2015) in SPM8, skull stripped (removing any extracerebral activity) and smoothed with a Gaussian kernel of $1.2 * 1.2 * 1.2$ full width at half-maximum (FWHM) to remove any non-uniformities. Finally, custom-made software was used to normalize the values of the ${ }^{18} \mathrm{~F}$ FDG uptake in each image to the average brain uptake value.

\section{Genes expression in VTA and NAc}

Rats from NSF and FUST experiment (Fig. 1B) were killed by decapitation $1 \mathrm{~h}$ after a single i.c.v. administration of GAL(1-15) $3 \mathrm{nmol}$ or vehicle and the brains were rapidly removed and frozen until its use. The nuclei dissections were conducted as described (Garcia-Perez et al., 2016) with modifications. Brains were sliced on the brain matrix $(1 \mathrm{~mm})$ and kept $-20^{\circ} \mathrm{C}$ until each region of interest comes into the cutting plane. For VTA analysis, one $1 \mathrm{~mm}$ coronal brain section was obtained from bregma -6.04 mm (Paxinos, 1986). For NAc study, two consecutive $1 \mathrm{~mm}$ coronal slices were made corresponding to approximate bregma $+1.2 \mathrm{~mm}$ (Paxinos, 1986). Tissues of interest were dissected using a punching device with $2 \mathrm{~mm}$ internal diameter. Bilateral punches of the NAc and VTA (Fig. 4 and 6) were collected into Eppendorf tubes.

\section{RNA isolation and quantitative real-time polymerase chain reaction analysis}

Total RNA was isolated from the punches of NAc and VTA using RNeasy Lipid Tissue Kit (Qiagen, Hilden, Germany). cDNA was obtained using a Reverse Transcriptase Core Kit (Eurogentec, Seraing, Belgium). These steps were performed according to the manufacturer's instructions. 
All PCR were conducted in triplicate using FastStart essential DNA green master (Roche Diagnostics $\mathrm{GmbH}$, Mannchem, Germany) in LigtCycler 96 system (Roche Diagnostics GmbH, Mannchem, Germany).

The data were analysed using the comparative $\mathrm{Ct}$ method and normalized to measures of glyceraldehyde- 3-phosphate dehydrogenase (GAPDH) mRNA.

\section{Tyrosine Hydroxylase immunohistochemistry}

Rats from NSF and FUST experiment (Fig. 1B) were perfused trascardially 90 min after a single i.c.v. administration of $\mathrm{GAL}(1-15) 3 \mathrm{nmol}$ or vehicle. The brains were then dissected and serial, coronal free-floating sections (30 $\mu \mathrm{m}$ thick) were collected. The sections were incubated with a primary antibody rabbit anti-TH (Sigma, 1/2000) overnight at $4^{\circ} \mathrm{C}$. An hour incubation with biotinylated, specific secondary antibodies (1:200; Vector Labs Inc) was performed at room temperature. The chromogen used was 0.03\% 3-30diaminobenzidine tetrahydrochloride (Sigma).

Analysis of TH Immunoreactivity was performed under light microscopy (Nikon Optiphot-2) in the neuro-anatomical area of interest. Three sections per animal were examined and captured by a video camera (Olimpus UC30) linked to a PC computer, and TH immunoreactivity was quantified by optical density using the computer software ImageJ (National Institutes of Health).

\section{Double Immunofluorescence}

The procedures have been previously used (Millon et al., 2015). Primary antibody rabbit anti-GAL2 receptor (Alomone Lab, 1/250) was incubated for 12 hours at $4^{\circ} \mathrm{C}$ and detected with the red secondary antibody mouse anti-rabbit DyLight 549 (Jackson inmunoResearch Laboratories, 1/100). Goat anti-GAL1 receptor (Santa Cruz Biotechnology INC, EEUU, 1:250) was incubated 12 hours at $4^{\circ} \mathrm{C}$ and detected with the secondary antibody rabbit biotynilated anti-goat (Vector Labs Inc.) and Alexa Fluor 488-conjugated Streptavidin (Jackson Laboratories InmunoResearch, 1:1000). Sections were mounted on slides with fluorescent mounting medium (Dako) and visualized using a Leica SP8 confocal microscope. The double immunolabeling was performed in the tissue of rats without treatment (Figure S1). 
Table S1. Oligonucleotide primers used in qPCR.

\begin{tabular}{clll}
\hline Gene & $\begin{array}{c}\text { Accession } \\
\text { number }\end{array}$ & \multicolumn{1}{c}{ Forward primer (5'-3') } & \multicolumn{1}{c}{ Reverse primer (5'-3') } \\
\hline Dat & NM_012694.2 & ACGGTGGCATCTACGTCTTC & TCACTGAATTGCTGGACGCC \\
Vmat2 & NM_013031.1 & GGATGGGGCTATTCAGCTCTT & AAAGCAGATGGAGCCTGCA \\
C-Fos & NM_022197.2 & AAACGGAGAATCCGAAGG & CGTCTTCAAGTTGATCTGTC \\
Drd1 & NM_012546.3 & AAACAAATAGGCCAAGAGTC & CTTTCCAATCTGAAAACTGC \\
Drd2 & NM_012547.1 & AAGCGCCGAGTTACTGTCAT & GACCACAAAGGCAGGGTTG \\
Drd3 & NM_017140.2 & CATCTACATAGTCCTGAGGC & CTGGCCCTTATTGAAAACTG \\
Drd5 & NM_012768.1 & AACTGTATGGTTCCTTTCTG & AATATGTCGAAGGTGGTCTC \\
Galr1 & NM_012958.3 & AAAACTGGACAAAACTTAGCC & GGATACCTTTGTCTTTGCTC \\
Galr2 & NM_019172.5 & AACAGGAATCCACAGACC & CCCTTTGGTCCTTTAACAAG \\
Gapdh & NM_017008.4 & GCTCTCTGCTCCTCCCTGTTC & GAGGCTGGCACTGCACAA \\
& & &
\end{tabular}

Table S2. Water intake $(\mathrm{g} / \mathrm{Kg})$ in Sucrose Preference Test.

\begin{tabular}{cccc}
\hline Treatment & $\mathbf{2 h}$ & $\mathbf{8 h}$ & $\mathbf{2 4 h}$ \\
\hline Control & $3.13 \pm 0.77$ & $7.46 \pm 1.49$ & $9.97 \pm 1.64$ \\
GAL(1-15) $3 \mathrm{nmol}$ & $8.55 \pm 2.58$ & $13.47 \pm 3.12$ & $16.58 \pm 2.90$ \\
\hline
\end{tabular}

Table S2. Effect of administration of Galanin (1-15) [GAL(1-15)] in the water intake in Sucrose Preference Test with $1 \%$ sucrose concentration in rats. GAL(1-15) (3nmol/rats; $\mathrm{n}=8-9$ animals per group) were administrated intracerebroventricular 2, 8 and 24 hours before the measures. Cerebrospinal fluid-injected rats were used as the Control group ( $n=7$ animals). Data represent the mean \pm standard error of the mean of water intake $(\mathrm{g} / \mathrm{Kg})$. No significant differences were found by repeated measures two-way ANOVA. 
Millón et al., 2018

\section{Journal of Psychopharmacology}

Figure S1

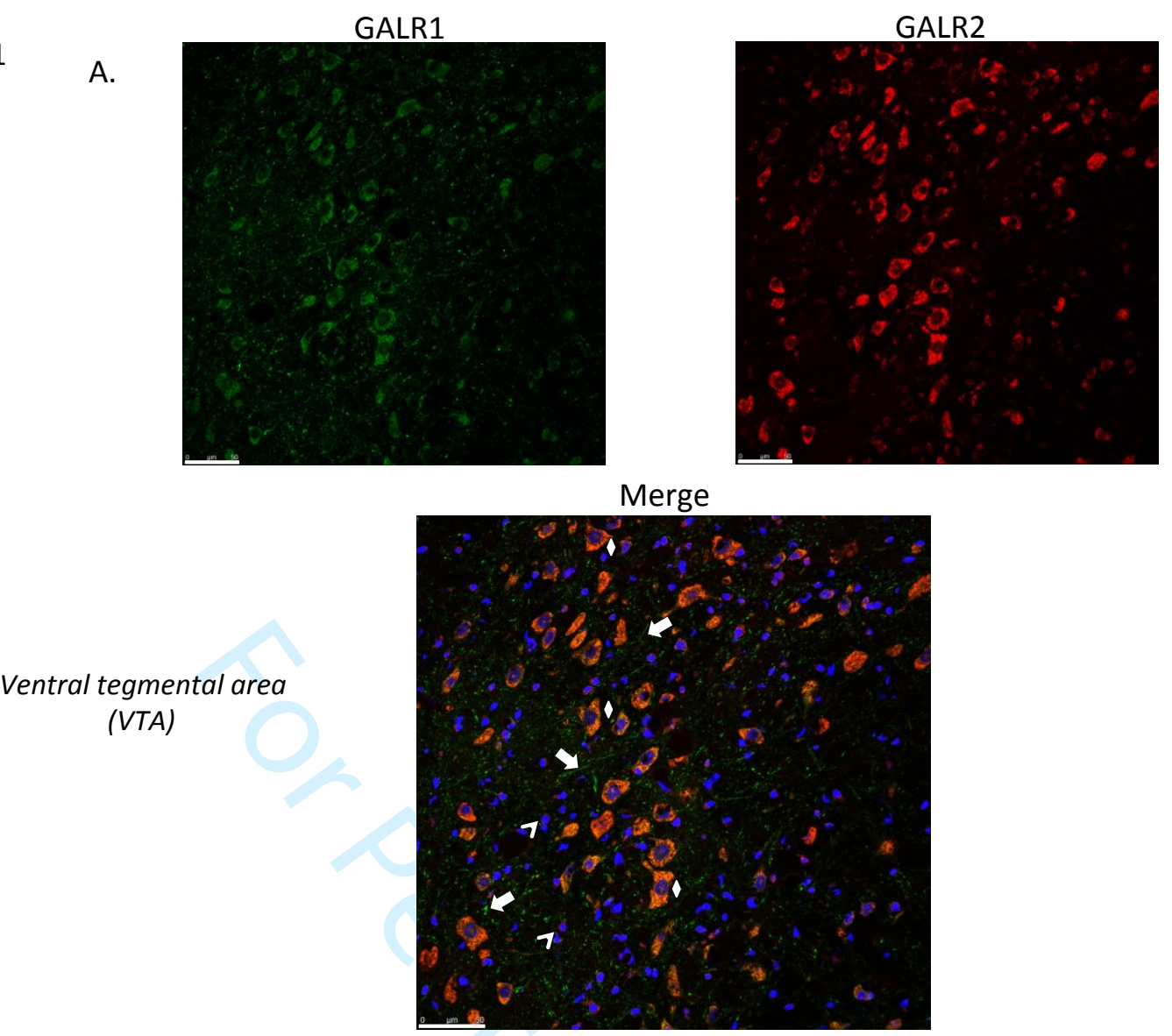

GALR1

B.

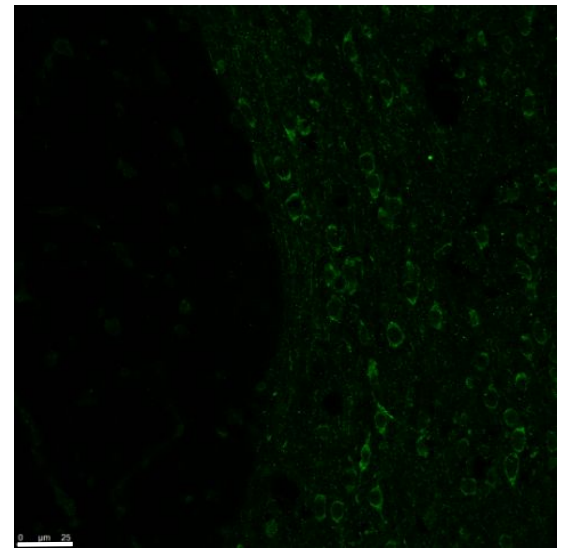

GALR2

\section{Accumbens nucleus Core (NAcC)}

Merge

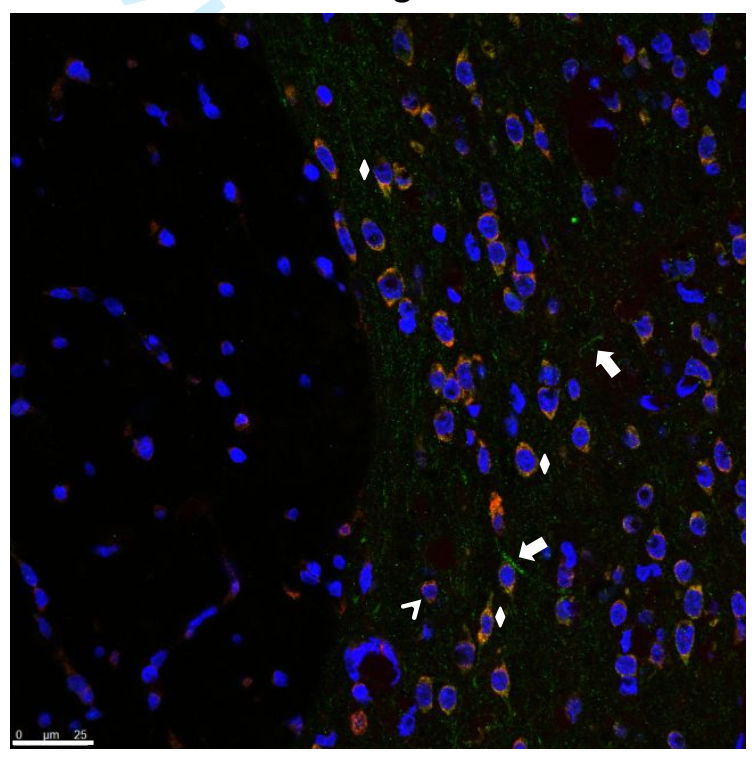




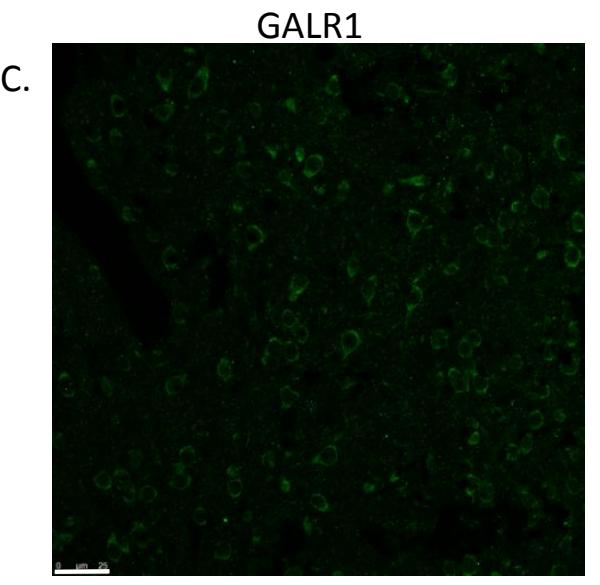

GALR2

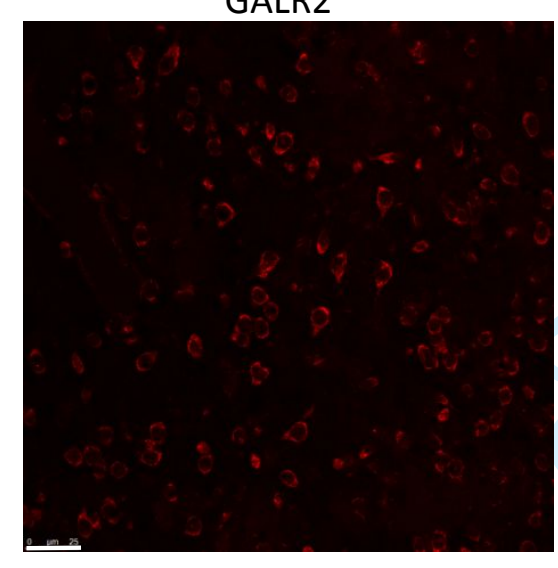

Caudate Putamen (CPu)

D.

GALR2
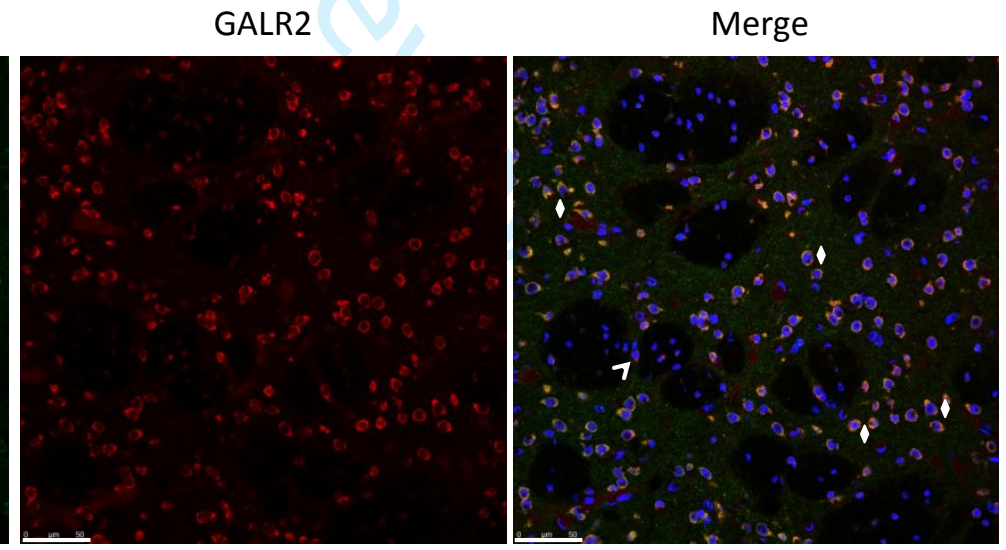

Figure S1. Galanin Receptor 1 (GALR1) and Galanin Receptor 2 (GALR2) colocalizes in neurons of rat striatum and Ventral Tegmental Area. Doubleimmunolabeled for GALR1 (green, arrows) and GALR2 (red, arrowhead) in (A) Ventral Tegmental Area (VTA), (B) Accumbens nucleus Core (NAcC), (C) Accumbens nucleus Shell (NAcSh) and (D) Caudate Putamen (Cpu). The colocalization of GALR1 and GALR2 is shown in the merge (orange-yellow, rhombus. Escale bar, 25-50 $\mu \mathrm{m}$. 


\section{REFERENCES}

(UMCG). NMaMI. (2015) Nuclear Medicine and Molecular Imaging (UMCG). SAMIT Introduction.

Alen F, Santos A, Moreno-Sanz G, et al. (2009) Cannabinoid-induced increase in relapse-like drinking is prevented by the blockade of the glycine-binding site of N-methyl-D-aspartate receptors. Neuroscience 158(2): 465-473.

Diaz-Cabiale Z, Parrado C, Narvaez M, et al. (2011) Galanin receptor/Neuropeptide $Y$ receptor interactions in the dorsal raphe nucleus of the rat. Neuropharmacology 61(1-2): 80-86.

Ferres-Coy A, Galofre M, Pilar-Cuellar F, et al. (2016) Therapeutic antidepressant potential of a conjugated siRNA silencing the serotonin transporter after intranasal administration. Mol Psychiatry 21(3): 328-338.

Garcia-Perez D, Nunez C, Laorden ML, et al. (2016) Regulation of dopaminergic markers expression in response to acute and chronic morphine and to morphine withdrawal. Addict Biol 21(2): 374-386.

Malkesman O, Austin DR, Tragon T, et al. (2012) Acute D-serine treatment produces antidepressant-like effects in rodents. Int J Neuropsychopharmacol 15(8): 1135-1148.

Marco EM, Ballesta JA, Irala C, et al. (2017) Sex-dependent influence of chronic mild stress (CMS) on voluntary alcohol consumption; study of neurobiological consequences. Pharmacol Biochem Behav 152: 68-80.

Millon C, Flores-Burgess A, Narvaez M, et al. (2015) A role for galanin N-terminal fragment (1-15) in anxiety- and depression-related behaviors in rats. Int J Neuropsychopharmacol 18(3): 1-13.

Pascual-Brazo J, Castro E, Diaz A, et al. (2012) Modulation of neuroplasticity pathways and antidepressant-like behavioural responses following the short-term (3 and 7 days) administration of the 5-HT(4) receptor agonist RS67333. Int J Neuropsychopharmacol 15(5): 631-643.

Paxinos G. (1986) The rat Brain in the stereotaxic coodinates.

Prieto E, Collantes M, Delgado M, et al. (2011) Statistical parametric maps of (1)(8)F-FDG PET and 3-D autoradiography in the rat brain: a crossvalidation study. Eur J Nucl Med Mol Imaging 38(12): 2228-2237.

Schwarz AJ, Danckaert A, Reese T, et al. (2006) A stereotaxic MRI template set for the rat brain with tissue class distribution maps and co-registered anatomical atlas: application to pharmacological MRI. Neuroimage 32(2): 538-550.

Technologies P. (2016) PMOD Image Fusion (PFUS). Version 3.8 ed.

Vallez Garcia D, Casteels C, Schwarz AJ, et al. (2015) A standardized method for the construction of tracer specific PET and SPECT rat brain templates: validation and implementation of a toolbox. PLoS One 10(3): e0122363. 